\title{
MULTIPLE STELLAR POPULATIONS IN 47 Tucanae*
}

\author{
A. P. Milone ${ }^{1,2}$, G. Piotto ${ }^{3}$, L. R. Bedin ${ }^{4}$, I. R. $\mathrm{King}^{5}$, J. Anderson ${ }^{4}$, A. F. Marino ${ }^{6}$, A. Bellini ${ }^{3}$, R. Gratton ${ }^{7}$, A. RenZini ${ }^{7}$,

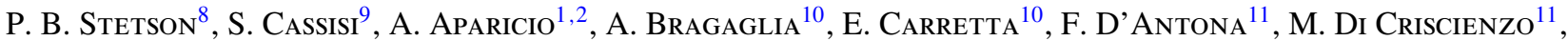 \\ S. LuCATEllo ${ }^{7}$, M. Monelli ${ }^{1,2}$, ANd A. Pietrinferni ${ }^{9}$ \\ ${ }^{1}$ Instituto de Astrofissica de Canarias, E-38200 La Laguna, Tenerife, Canary Islands, Spain; milone@iac.es, aparicio@iac.es, monelli@iac.es \\ ${ }^{2}$ Department of Astrophysics, University of La Laguna, E-38200 La Laguna, Tenerife, Canary Islands, Spain \\ ${ }^{3}$ Dipartimento di Astronomia, Università di Padova, Padova I-35122, Italy; giampaolo.piotto@unipd.it, andrea.bellini@unipd.it \\ ${ }^{4}$ Space Telescope Science Institute, Baltimore, MD 21218, USA; jayander@stsci.edu, bedin@ stsci.edu \\ ${ }^{5}$ Department of Astronomy, University of Washington, Seattle, WA 98195-1580, USA; king@ astro.washington.edu \\ ${ }^{6}$ Max-Planck-Institut für Astrophysík, D-85741 Garching, Germany; amarino@MPA-Garching.MPG.DE \\ ${ }^{7}$ INAF-Osservatorio Astronomico di Padova, I-35122 Padua, Italy; \\ raffaele.gratton@oapd.inaf.it, alvio.renzini@oapd.inaf.it, sara.lucatello@oapd.inaf.it \\ ${ }^{8}$ Dominion Astrophysical Observatory, Herzberg Institute of Astrophysics, National Research Council, \\ Victoria, BC V9E 2E7, Canada; Peter.Stetson@nrc-cnrc.gc.ca \\ ${ }^{9}$ INAF-Osservatorio Astronomico di Collurania, I-64100 Teramo, Italy; \\ cassisi@oa-teramo.inaf.it,pietrinferni@oa-teramo.inaf.it \\ ${ }^{10}$ INAF-Osservatorio Astronomico di Bologna, I-40127 Bologna, Italy; \\ angela.bragaglia@oabo.inaf.it, eugenio.carretta@oabo.inaf.it \\ ${ }^{11}$ INAF-Osservatorio Astronomico di Roma, I-00040 Monte Porzio Catone, Rome, Italy; \\ dantona@mporzio.astro.it, dicrisci@gmail.com \\ Received 2011 May 4; accepted 2011 September 3; published 2011 December 13
}

\begin{abstract}
We use Hubble Space Telescope (HST) and ground-based imaging to study the multiple populations of 47 Tucanae (47 Tuc), combining high-precision photometry with calculations of synthetic spectra. Using filters covering a wide range of wavelengths, our HST photometry splits the main sequence into two branches, and we find that this duality is repeated in the subgiant and red giant regions, and on the horizontal branch. We calculate theoretical stellar atmospheres for main-sequence stars, assuming different chemical composition mixtures, and we compare their predicted colors through the HST filters with our observed colors. We find that we can match the complex of observed colors with a pair of populations, one with primeval abundance and another with enhanced nitrogen and a small helium enhancement, but with depleted $\mathrm{C}$ and $\mathrm{O}$. We confirm that models of red giant and red horizontal branch stars with that pair of compositions also give colors that fit our observations. We suggest that the different strengths of molecular bands of $\mathrm{OH}, \mathrm{CN}, \mathrm{CH}$, and $\mathrm{NH}$, falling in different photometric bands, are responsible for the color splits of the two populations. Near the cluster center, in each portion of the color-magnitude diagram the population with primeval abundances makes up only $\sim 20 \%$ of the stars, a fraction that increases outward, approaching equality in the outskirts of the cluster, with a fraction $\sim 30 \%$ averaged over the whole cluster. Thus the second, He/N-enriched population is more concentrated and contributes the majority of the present-day stellar content of the cluster. We present evidence that the color-magnitude diagram of 47 Tuc consists of intertwined sequences of the two populations, whose separate identities can be followed continuously from the main sequence up to the red giant branch, and thence to the horizontal branch. A third population is visible only in the subgiant branch, where it includes $\sim 8 \%$ of the stars.
\end{abstract}

Key words: Hertzsprung-Russell and C-M diagrams - stars: abundances - stars: horizontal-branch - stars: Population II - techniques: photometric

Online-only material: color figures

\section{INTRODUCTION}

The presence of multiple stellar populations in globular clusters (GCs) has been widely established both by photometric and by spectroscopic studies. More than 40 years ago the red giant branch (RGB) of $\omega$ Centauri (NGC 5139) was found to have a photometric spread in color (Woolley 1966), associated with a metallicity spread (Freeman \& Rodgers 1975; Norris $\&$ Bessell 1975), but the first real challenge to the traditional picture of GCs as simple stellar populations came from the discovery of abundance anomalies among stars within the same GCs (e.g., Kraft 1979). All Galactic GCs studied so far show

\footnotetext{
* Based on observations with the NASA/ESA Hubble Space Telescope, obtained at the Space Telescope Science Institute, which is operated by AURA, Inc., under NASA contract NAS 5-26555.
}

$\mathrm{Na}-\mathrm{O}$ anticorrelations (e.g., Carretta et al. 2009b), indicative of contamination from products of proton-capture reactions at high temperature (Denisenkov \& Denisenkova 1989; Langer et al. 1993). Moreover, the presence of such anticorrelations in unevolved main-sequence (MS) stars (e.g., Gratton et al. 2001; Ramírez \& Cohen 2002), which have not yet reached sufficiently high temperatures in their interiors, suggests that more than one generation of stars has formed within Galactic GCs (see Gratton et al. 2004 for a review), possibly in combination with accretion onto low-mass stars of ejecta from either intermediate-mass stars (D'Antona et al. 1983; Renzini 1983), fast-rotating massive stars (e.g., Decressin et al. 2007), or massive binaries (De Mink et al. 2009).

Clear evidence of a complex star formation history in GCs has come from high-precision Hubble Space Telescope (HST) 
photometry, which showed unequivocally that the phenomenon of multiple sequences in the color-magnitude diagrams (CMDs) of GCs is not confined to the special case of $\omega$ Centauri (for which see, e.g., Anderson 1997; Bedin et al. 2004). Split or spread MSs have been observed in NGC 2808, 47 Tucanae (47 Tuc; NGC 104), and NGC 6752 (Piotto et al. 2007; Anderson et al. 2009; Milone et al. 2010), while splits in the subgiant branches (SGBs) have been detected in NGC 1851, NGC 6656 (M22), 47 Tuc, and at least five other GCs (Milone et al. 2008; Marino et al. 2009; Anderson et al. 2009; Piotto 2009). The splitting of the RGB has been observed in all the GCs studied to date with appropriate photometric bands (e.g., Marino et al. 2008; Yong et al. 2008; Lee et al. 2009; Lardo et al. 2011). The photometric investigations definitively confirm that it common for GCs to contain multiple stellar populations.

More recently, it has been possible to connect the photometrically observed multiple sequences with differences of chemical composition among the stars in the same cluster. The aim of such studies has been to understand how successive generations of stars could have formed in a GC, and what could be inferred about the nature of the polluters from their chemical imprint on the second-generation stars. The first notable results were by Piotto et al. (2005), who showed that the bluer MS in $\omega$ Centauri is more metal-rich than the redder one. The only way to reconcile the photometric and spectroscopic results is to assume that the bluer MS is strongly He-enhanced. Other examples are given by Marino et al. (2008), who showed that the presence of two groups of stars with different $\mathrm{C}, \mathrm{N}, \mathrm{Na}$, and $\mathrm{O}$ content is at the root of the difference in the $(U-B)$ color of the RGB stars in NGC 6121 (M4). Na-poor (CN-weak) giants define a sequence bluer than the one occupied by the Na-rich ( $\mathrm{CN}$-strong) ones. Yong et al. (2008) found that RGB spreads are present in a large number of GCs, when using the Strömgren $c 1$ index, which is a powerful tracer of the $\mathrm{N}$ abundance. In general, stellar evolutionary models suggest that observed multiple MSs should correspond to stellar populations with different He abundance, the bluer sequences having a higher helium abundance than primordial (e.g., Norris 2004; D'Antona et al. 2005; Piotto et al. 2005, 2007; Di Criscienzo et al. 2010, 2011). According to this picture, the triple MS in NGC 2808 can also correspond to the three groups of stars with different oxygen and sodium abundances observed among the RGB stars (Carretta et al. 2006), likely to come from three successive episodes of star formation. In fact, hydrogen burning at high temperatures through the $\mathrm{CNO}$ cycle and subsequent proton captures result in an $\mathrm{He}$ enrichment, and at the same time in an enhancement of $\mathrm{N}, \mathrm{Na}$, and $\mathrm{Al}$, and a depletion of $\mathrm{C}, \mathrm{O}$, and $\mathrm{Mg}$. This scenario has been nicely confirmed for NGC 2808 by Bragaglia et al. (2010), who measured chemical abundances of one star on the red MS and one on the blue MS, and found that the latter shows an enhancement of $\mathrm{N}, \mathrm{Na}$, and $\mathrm{Al}$, and a depletion in $\mathrm{C}$ and $\mathrm{Mg}$. To date there are no spectroscopic studies of stars on the middle branch of the MS, however.

Finally, theoretical models have proposed that the SGB split observed in some GCs could be due to two stellar groups with either an age difference of 1-2 Gyr or a different $\mathrm{C}+\mathrm{N}+\mathrm{O}$ content (Cassisi et al. 2008; Ventura et al. 2009). In support of the chemical-content scenario, a bimodality in the $s$-process elements and in the CNO has been detected in NGC 1851 and M22 (Yong et al. 2009; Marino et al. 2011b). From the theoretical point of view, a unique investigation of the impact that the chemical patterns observed in GC sub-populations have on the CMD comes from Sbordone et al. (2011).
Summarizing the observational and theoretical scenarios:

1. split MSs suggest helium enrichment and also correspond to different groups of stars in the $\mathrm{Na}-\mathrm{O}$ anticorrelation;

2. multiple SGBs might be ascribed to different total abundance of $\mathrm{C}+\mathrm{N}+\mathrm{O}$ or to different age;

3. photometrically multiple RGBs might be due to differences in $\mathrm{C}, \mathrm{N}, \mathrm{O}$ content via the different strengths of the corresponding molecular features; and

4. there appears to be no strong indication of any significant spread in $[\mathrm{Fe} / \mathrm{H}]$ (except for a few clusters: $\omega$ Centauri, M22, Terzan 5, and NGC 2419).

Multiple stellar populations with different helium abundance also offer an explanation for the complex, extended, and clumpy horizontal branch (HB) morphology exhibited by some clusters (e.g., D'Antona et al. 2005; D'Antona \& Caloi 2004; Catelan et al. 2010; Gratton et al. 2010). A direct confirmation of a connection of the HB shape with the chemical content of the HB comes from recent work by Marino et al. (2011a), who have found that stars on the blue side of the instability strip of the cluster M4 are Na-rich and O-poor, whereas stars on the red HB are all Na-poor.

While the presence of multiple stellar populations in GCs as revealed by multiplicities of the $\mathrm{MS}, \mathrm{SGB}, \mathrm{RGB}$, or $\mathrm{HB}$ has been clearly established in many clusters, efforts to unequivocally connect the various evolutionary stages of each individual stellar generation have met with only modest success so far, because individual sequences appear to merge and even cross each other in some parts of the CMD, strongly depending on the photometric bands used to build the CMD. Connecting the various branches from the MS to the HB would greatly help to fully characterize each individual stellar generation, in terms of composition and age.

In the present paper, we attack this problem by applying highprecision HST photometry to the GC 47 Tucanae (GO-12311, PI: G. Piotto). This is one of the GCs in the Milky Way where multiple stellar populations have recently been detected and studied both photometrically and spectroscopically. From the analysis of a large number of archival HST images of the inner $\sim 3 \times 3$ arcmin, Anderson et al. (2009) found that the SGB is spread in magnitude, with at least two distinct branches: a brighter one with an intrinsic broadening in luminosity and a second one about 0.05 mag fainter that includes a small fraction of the stars. Anderson et al. were also able to study the MS in a less crowded field 6 arcmin from the center and found an intrinsic broadening that increases toward fainter magnitudes. They interpreted the MS spread in terms of a variation of helium abundance of $\sim 0.02-0.03$. Di Criscienzo et al. (2010) suggested that a spread in helium of $\sim 0.02$ might be responsible for both the luminosity spread of the bright SGB and the HB morphology, whereas an increase in the overall $\mathrm{C}+\mathrm{N}+\mathrm{O}$ abundance could be responsible for the faint SGB. In substantial agreement with the above estimates of the differences in helium content in 47 Tuc, Nataf et al. (2011) have estimated a helium difference of $\Delta Y \simeq 0.03$ between two sub-populations of this cluster, based on the strength and luminosity of the RGB bump and on the luminosity of the HB. They also noted that the helium-rich population is more centrally concentrated.

Since the early seventies, spectroscopic investigations have shown that RGB stars in 47 Tuc exhibit large star-to-star variations in CN band strength (e.g., McClure \& Osborn 1974; Bell et al. 1975), with two distinct groups of stars showing different CN content (Norris \& Freeman 1979; Briley 1997); this 
Table 1

HST Data Sets Used in This Paper

\begin{tabular}{|c|c|c|c|c|c|}
\hline Instr & Date & $N \times$ EXP TIME & Filter & Program & PI \\
\hline UVIS/WFC3 & 2011 Nov 21-22 & $2 \times 323 s+12 \times 348 s$ & F275W & 12311 & Piotto \\
\hline UVIS/WFC3 & 2010 Sep 28 & $30 s+1160 s$ & F336W & 11729 & Holtzman \\
\hline UVIS/WFC3 & 2010 Sep 28 & $2 \times 10 s+2 \times 348 s+2 \times 940 s$ & F390W & 11664 & Brown \\
\hline $\mathrm{ACS} / \mathrm{WFC}$ & 2002 Sep 30 and Oct 11 & $9 \times 105 \mathrm{~s}$ & F435W & 9281 & Grindlay \\
\hline $\mathrm{ACS} / \mathrm{WFC}$ & 2002 Apr 5 & $20 \times 60 \mathrm{~s}$ & F475W & 9028 & Meurer \\
\hline $\mathrm{ACS} / \mathrm{WFC}$ & 2002 Jul 7 & $5 \times 60 \mathrm{~s}$ & F475W & 9443 & King \\
\hline $\mathrm{ACS} / \mathrm{WFC}$ & $2002 \mathrm{Jul} 7$ & $1 \times 150 \mathrm{~s}$ & F555W & 9443 & King \\
\hline $\mathrm{ACS} / \mathrm{WFC}$ & 2006 Mar 13 & $3 s+4 \times 50 s$ & F606W & 10775 & Sarajedini \\
\hline $\mathrm{ACS} / \mathrm{WFC}$ & 2002 Sep 30 and Oct 11 & $20 \times 65 s$ & F625W & 9281 & Grindlay \\
\hline $\mathrm{ACS} / \mathrm{WFC}$ & 2006 Mar 13 & $3 s+4 \times 50 s$ & F814W & 10775 & Sarajedini \\
\hline
\end{tabular}

dichotomy is also present among MS stars (Cannon et al. 1998; Harbeck et al. 2003). An Na-O anticorrelation has recently been studied by Carretta et al. (2009a, 2009b), with 30\% of the stars being Na-poor and O-rich, while the remaining $~ 70 \%$ are depleted in oxygen and enhanced in sodium.

This paper is organized as follows. In Section 2, we describe the data and the reduction. Section 3 reveals a split in the MS, and in Section 4 we explore possible theoretical interpretations. Sections 5-7 return to the pursuit of multiple sequences along the SGB, the RGB, and the HB, respectively, and also explore their interpretation. The spatial distribution of multiple stellar populations is investigated in Section 8, while in Section 9 we attempt to connect the multiple sequences that we have found along the MS, the SGB, the RGB, and the HB, and to trace the CMD of each of the two stellar generations. A summary and some final discussion follow in Section 10.

\section{OBSERVATIONS AND DATA REDUCTION}

For our study of the stellar populations in 47 Tuc, we used data sets from two different telescopes. For the crowded central regions of the cluster we used $H S T$ images taken with the Wide Field Channel of the Advanced Camera for Surveys (ACS/WFC) and the UVIS channel of Wide Field Camera 3 (WFC3/UVIS), while to study the spatial distribution of the populations we made use of $U, B, V$, and $I$ ground-based photometry from the data base of 856 original and archival CCD images from Stetson (2000). Among them, 480 images were obtained with the Wide-Field Imager of the ESO/MPI $2.2 \mathrm{~m}$ telescope, and 200 with the $1.5 \mathrm{~m}$ telescope at Cerro Tololo Inter-American Observatory, while the remaining 176 images come from various other telescopes. These observations are described in detail in Bergbusch \& Stetson (2009). They were reduced following the protocol outlined in some detail by Stetson (2005) and are calibrated on the Landolt (1992) photometric system.

Table 1 summarizes the characteristics of the HST images that we used, while Figure 1 shows their footprints. The ACS images, and a number of the WFC3 images, were archival, except that the images of GO-12311 (PI: G. Piotto) were taken expressly for this project, and were crucial to its success.

The ACS/WFC images were reduced by using the software described in Anderson et al. (2008). It consists of a package that analyzes all the exposures simultaneously to generate a catalog of stars over the whole field of view. Stars are measured in each image independently by using for each filter a spatially variable point-spread-function model from Anderson \& King (2006) plus a "perturbation PSF" that allows for the effects of focus variations. The photometry was put into the Vega-mag

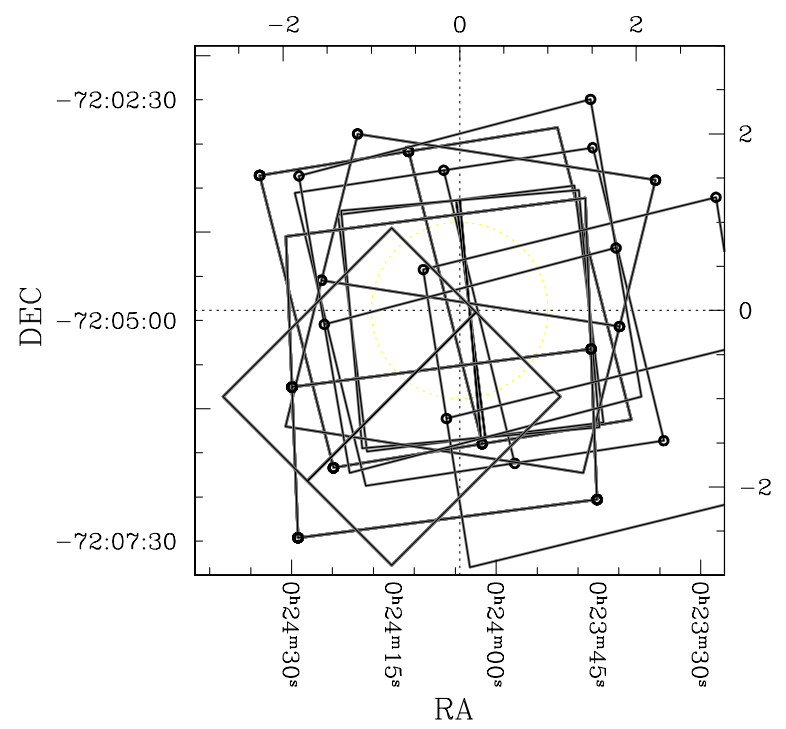

Figure 1. Footprints of our HST fields. The small circles are the corners of ACS/WFC chip 1. The footprints of WFC3/UVIS exposures are distinguished by having no such marking.

system following the recipes of Bedin et al. (2005) and using the encircled energy and zero points of Sirianni et al. (2005).

Star positions and fluxes in the WFC3 images were measured with software that is mostly based on img2xym_WFI (Anderson et al. 2006); this will be presented in a separate paper. Star positions and fluxes were corrected for pixel area and geometric distortion by using the solution given by Bellini \& Bedin (2010) and Bellini et al. (2011), and were calibrated as in Bedin et al. (2005).

The work that we present here is based mainly on highprecision photometry, for which our next step was to select a high-quality sample of stars that are relatively isolated and have small photometric and astrometric errors, and are also well fit by the PSF. For this we used the quality indices that our photometry software produces, in a procedure that is described in detail by Milone et al. (2009, Section 2.1). Finally, we corrected our photometry for some remaining position-dependent errors, due to small inadequacies in our PSFs that were quite small but were different for each filter. Since all the uses of our photometry would depend on colors, we generated the 36 colors that can be derived from our nine filters. For each of these colors we drew the MS ridgeline in the corresponding CMD; then for each star we identified its 50 closest well-measured neighbors and found their median-color offset from the MS ridgeline. Since this constituted a good estimate of the systematic color error at 


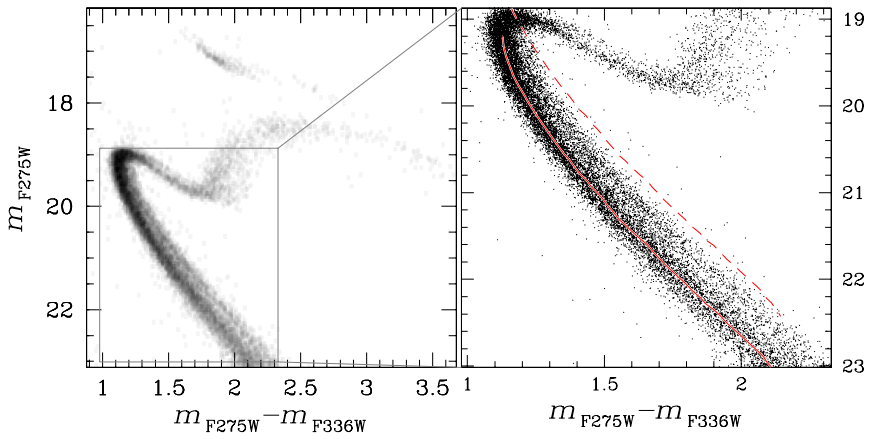

Figure 2. $m_{\mathrm{F} 275 \mathrm{~W}}$ vs. $m_{\mathrm{F} 275 \mathrm{~W}}-m_{\mathrm{F} 336 \mathrm{~W}}$ Hess diagram (left) and CMD zoomed around the MS region (right). The continuous and the dashed red lines in the right panel mark the MS ridgeline of MSb and the equal-mass binary sequence, respectively.

(A color version of this figure is available in the online journal.)

the position of the target star, for that pair of filters we corrected the observed color of the star by that amount.

\section{THE DOUBLE MAIN SEQUENCE}

As already mentioned in Section 1, the initial evidence that the MS of 47 Tuc is not consistent with a single stellar population comes from the recent work by Anderson et al. (2009), who detected an intrinsic spread in the $m_{\mathrm{F} 606 \mathrm{~W}}-$ $m_{\mathrm{F} 814 \mathrm{~W}}$ color ranging from $\sim 0.01$ mag near $m_{\mathrm{F} 606 \mathrm{~W}} \sim 19.0$ to $\sim 0.02 \mathrm{mag}$ around $m_{\mathrm{F} 606 \mathrm{~W}}=22.0$. Our present data set, however, allowed us to study the MS with higher precision than was possible for Anderson et al. with the data available at that time.

An inspection of the large number of CMDs that we obtain from the data set listed in Table 1 showed that the multiple populations along the MS are best recognized and separated from photometry that combines $m_{\mathrm{F} 275 \mathrm{~W}}$ with $m_{\mathrm{F} 336 \mathrm{~W}}$. The lefthand panel of Figure 2 shows the Hess diagram of $m_{\mathrm{F} 275 \mathrm{~W}}$ versus $m_{\mathrm{F} 275 \mathrm{~W}}-m_{\mathrm{F} 336 \mathrm{~W}}$, after the quality selection and photometric corrections described in the previous section. We immediately note a widely spread RGB, a bimodal SGB, and a double MS. To examine these more closely, in the right-hand half of the figure we show a CMD that is zoomed around the upper MS, the SGB, and the start of the RGB. We defer discussion of the SGB and RGB to later sections and concentrate here on the MS morphology.

The CMD in the right-hand panel suggests that the MS of 47 Tuc is bimodal, in analogy with the multiple MSs observed in $\omega$ Centauri, NGC 2808, and NGC 6752. In 47 Tuc, however, the majority of MS stars populate the blue component (hereafter $\mathrm{MSb}$ ), while a small but significant fraction of the stars lie on a redder MS branch (MSa). The two sequences merge close to the turnoff, but on the MS the separation of the two components increases toward fainter magnitudes, from $0.08 \mathrm{mag}$ at $m_{\mathrm{F} 275 \mathrm{~W}}=19.5$ to $0.15 \mathrm{mag}$ at $m_{\mathrm{F} 275 \mathrm{~W}}=23$ - as illustrated in more detail in Figure 3. Such large, clear separations allow us to exclude any possibility that the MS split might be due to measuring errors.

We note here that the "verticalizing" of the MS in Figure 3 is a process that we will carry out several times in the course of this
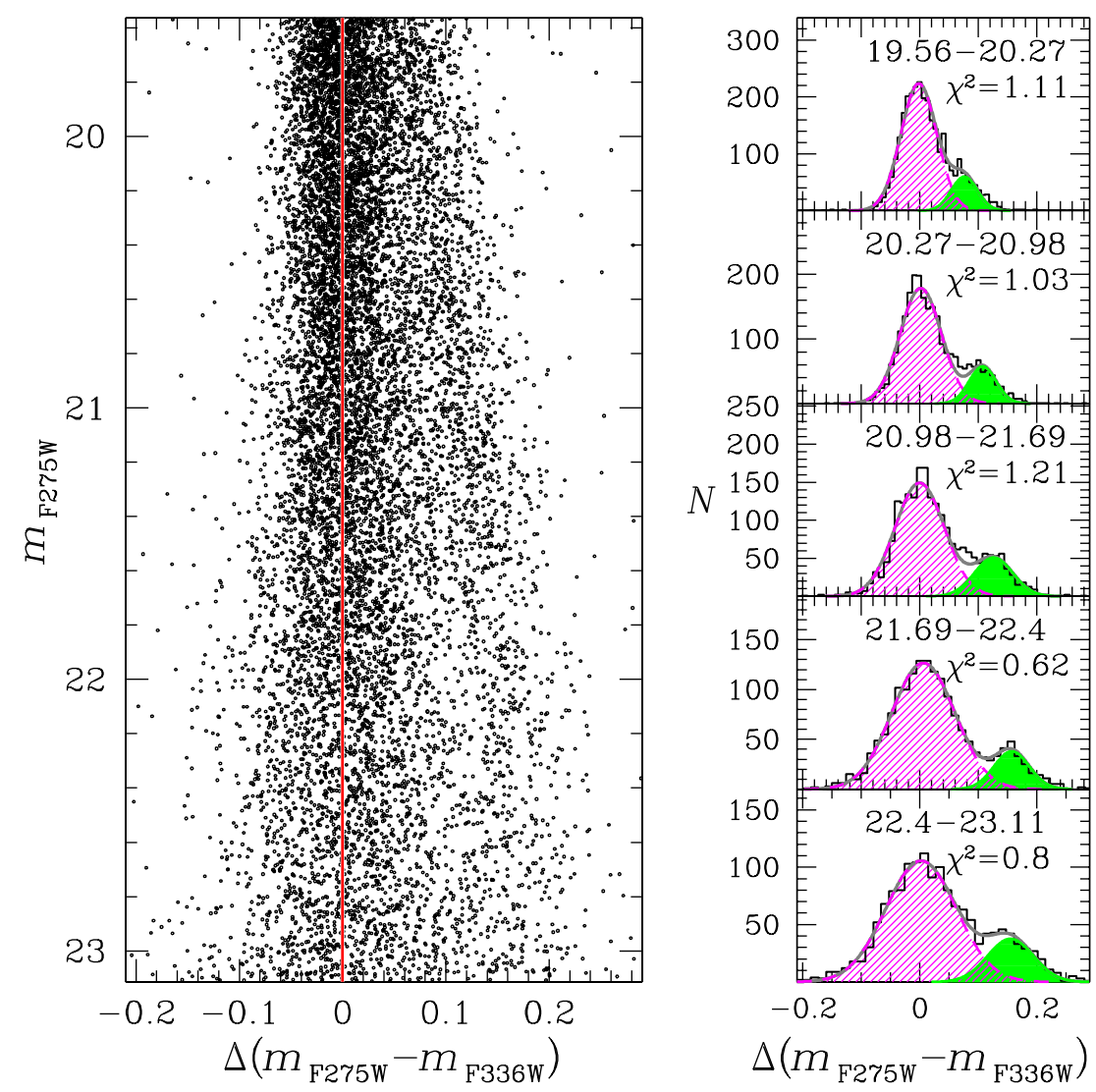

Figure 3. Left: the same CMD as in Figure 2, but after subtracting from the color of the MS the color of the ridgeline of MSb. Right: color distributions of the points in the left panel, showing two clear peaks. The magenta and green solid lines are the least-squares fits of two Gaussians to the histograms, while the gray line is their sum. We have also indicated the reduced- $\chi^{2}$ value corresponding to each bi-Gaussian fit.

(A color version of this figure is available in the online journal.) 


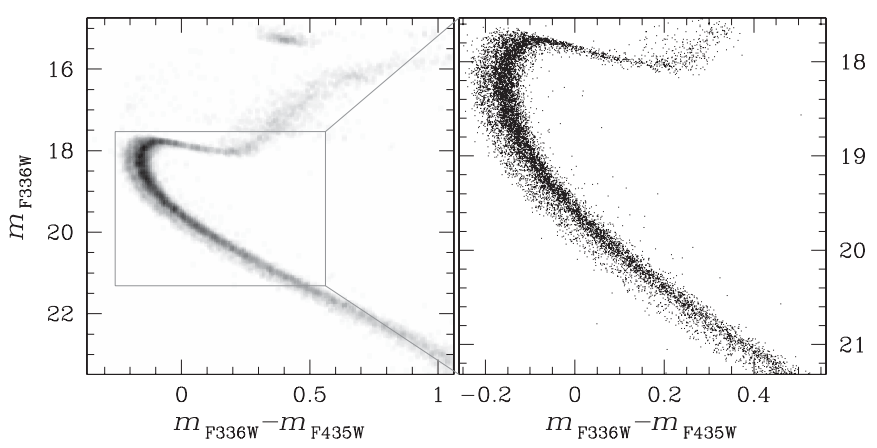

Figure 4. $m_{\mathrm{F} 336 \mathrm{~W}}$ vs. $m_{\mathrm{F} 336 \mathrm{~W}}-m_{\mathrm{F} 435 \mathrm{~W}}$ Hess diagram (left panel) and CMD zoomed around the region where the split is most evident (right panel). Note that, contrary to its behavior in the CMD of Figure 2, the less populous MS is bluer here than the bulk of MS stars.

paper and now explain it once and for all. We first designated $\mathrm{MSb}$ as our target sequence, by means of a hand-drawn first approximation to its ridgeline, and we also chose a limited color range around this line. We then put a spline through the median colors in successive short intervals of magnitude and did an iterated sigma clipping of outliers; the result was a fiducial sequence for MSb. The verticalization then consisted of subtracting from the color of each star the color of the fiducial sequence at the magnitude of that star.

Figure 3 allows us to estimate the fractions of MSa and MSb stars by assigning to each star a verticalized color (left panel of the figure), as explained above. The right-hand panels of the figure show the color histograms for five magnitude bins; in each bin we fitted the histogram with a pair of Gaussians, shown in magenta and green. These colors will be used consistently hereafter to distinguish these two sequences and their post-MS progeny. From the areas under the Gaussians we estimate that $82 \%$ of the stars belong to MSb and $18 \%$ to $\mathrm{MSa}$; within the statistical uncertainties of these numbers they have the same values in each of the magnitude intervals.

We also note that MSa cannot be ascribed to a sequence of binaries. The dashed line in Figure 2 is the equal-mass-binary sequence that corresponds to the fiducial sequence of MSb. Interpreting the stars of MSa as binaries would require making the outlandish hypothesis that about a fourth of the MS stars in 47 Tuc are in binary systems with mass ratio in the narrow interval 0.7-0.8. In addition, assuming that all MSa stars are binaries would imply a binary fraction $(\geqslant 15 \%)$, in sharp contrast with recent estimates of a $2 \%$ binary fraction by Milone et al. (2008a, 2008 b). ${ }^{12}$ In view of this, we expect that binaries do not affect the following discussion in any significant way.

The double MS is also evident in CMDs that use a different combination of magnitude and color, as shown in the $m_{\mathrm{F} 336 \mathrm{~W}}$ versus $m_{\mathrm{F} 336 \mathrm{~W}}-m_{\mathrm{F} 435 \mathrm{~W}}$ Hess diagram and CMD of Figure 4 . It is important to note that in this color system the less populous MS component is bluer than the other MS stars.

Since these two CMDs from the filter set F275W, F336W, and $\mathrm{F} 435 \mathrm{~W}$ behave so differently, we construct the two-color diagram that is shown on the left side of Figure 5. There is a clear separation between the two populations, and in the right panel the stars on opposite sides of the dividing line have been colored green and magenta to identify stars of MSa and MSb, respectively.

\footnotetext{
12 We note that the larger binary fraction for 47 Tuc proposed by Albrow et al (2001) comes from extrapolation from the fraction of W UMa stars that they found in the same cluster, based on assumptions about the distribution of binary periods, and W UMa binary evolution.
}

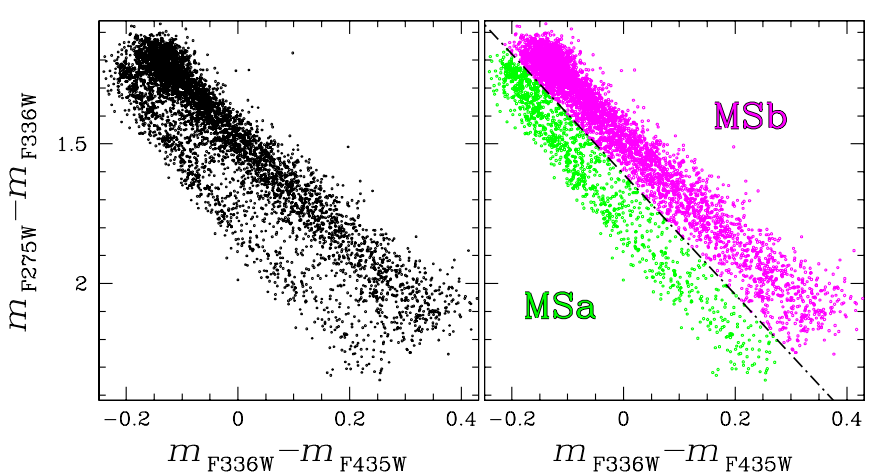

Figure 5. $m_{\mathrm{F} 275 \mathrm{~W}}-m_{\mathrm{F} 336 \mathrm{~W}}$ vs. $m_{\mathrm{F} 336 \mathrm{~W}}-m_{\mathrm{F} 435 \mathrm{~W}}$ two-color diagram for MS stars with $19.56<m_{\mathrm{F} 275 \mathrm{~W}}<23.11$. The dot-dashed line in the right-hand panel separates the MSa and MSb stars, shown in green and magenta colors, respectively.

(A color version of this figure is available in the online journal.)

With high-accuracy photometric measurements in nine bands, 36 different CMDs could be generated, if we were to use all possible combinations of magnitude and color.

In the upper panels of Figure 6, we show three of the most representative of these CMDs, zoomed around the MS region, with each star colored green or magenta, according to its membership in MSa or MSb as shown in Figure 5. In the bottom panels of Figure 6 the same CMDs are replicated and superimposed on the observed CMDs are the fiducial ridgelines of $\mathrm{MSa}$ and $\mathrm{MSb}$, derived for each CMD using the method described above.

The fiducials for the two MSs are then shown again in Figure $7\left(m_{\mathrm{F} 275 \mathrm{~W}}\right.$ versus $\left.m_{\mathrm{F} 275 \mathrm{~W}}-m_{\mathrm{X}}\right)$, Figure $8\left(m_{\mathrm{F} 336 \mathrm{~W}}\right.$ versus $m_{\mathrm{F} 336 \mathrm{~W}}-m_{\mathrm{X}}$ or $\left.m_{\mathrm{X}}-m_{\mathrm{F} 336 \mathrm{~W}}\right)$, and Figure $9\left(m_{\mathrm{F} 814 \mathrm{~W}}\right.$ versus $m_{\mathrm{X}}-m_{\mathrm{F} 814 \mathrm{~W}}$ ), where $m_{\mathrm{X}}$ is indicated on the ordinate of each of row of figures. We note that $\mathrm{MSa}$ is redder than $\mathrm{MSb}$ in most of these CMDs, but the color ordering of the two fiducial lines is otherwise in some of them: MSa stars become bluer than MSb stars in the $m_{\mathrm{F} 336 \mathrm{~W}}-m_{\mathrm{X}}$ colors (Figure 6, lower right panel, and Figure 8).

\section{INTERPRETING THE TWO BRANCHES OF THE MAIN SEQUENCE}

Surely the bizarre behavior of the two branches of the MS in our various CMDs is telling us something about the physical origin of the split. In hopes of clarifying the complicated observational picture, we will focus on a critical subset of the data, then we will compare the observations against synthetic spectra that have been calculated for atmospheres with various chemical compositions.

The challenge of choosing a critical subset from our mass of observational results is similar to the one faced by Bellini et al. (2010) in their multicolor study of $\omega$ Centauri; we follow their lead and look at the separation of the sequences as a function of the color index in which they are observed. Figure 10 shows the separation of MSa and MSb in each of eight colors; the figure also compares these observational separations of the sequences with calculated separations, from three theoretical scenarios that we will describe below.

On the theoretical side, multiplicity of MSs has been attributed to differences in helium content (e.g., Norris 2004; D'Antona et al. 2005; Piotto et al. 2005, 2007) or in lightelement abundances (e.g., Sbordone et al. 2011), so we will explore both of these possibilities by making three different 

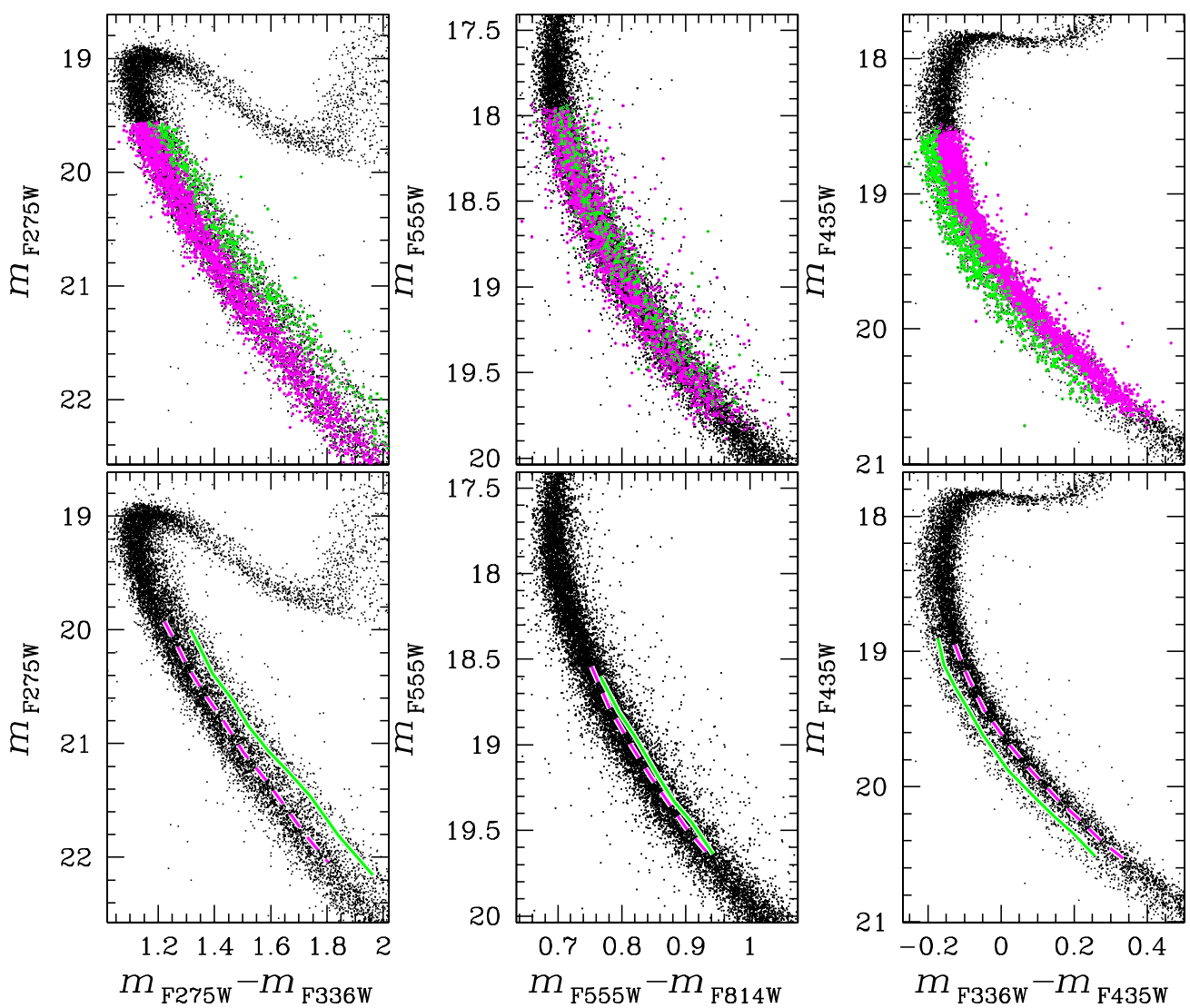

Figure 6. Example of the definition of MS fiducials. Upper panels show three different CMDs zoomed around the MS, with the MSa and MSb stars defined in Figure 5 plotted in green and magenta colors, respectively. In the lower panels we have superposed on these CMDs the MSa fiducial line (green continuous line) and the MSb fiducial (magenta dashed line), calculated in each case by the median-color, spline, sigma-clip procedure that was described earlier in the text.

(A color version of this figure is available in the online journal.)

Table 2

Parameters Used to Simulate Synthetic Spectra of an MSa and an MSb Star with $m_{\mathrm{F} 814 \mathrm{~W}}=18.2$, for the Three Assumed Options

\begin{tabular}{lcccrcr}
\hline \hline MS (Option) & $T_{\text {eff }}$ & $\log g$ & $Y$ & {$[\mathrm{C} / \mathrm{Fe}]$} & {$[\mathrm{N} / \mathrm{Fe}]$} & {$[\mathrm{O} / \mathrm{Fe}]$} \\
\hline MSa (all) & 5563 & 5.42 & 0.256 & 0.06 & 0.20 & 0.40 \\
MSb (I) & 5648 & 5.41 & 0.288 & 0.06 & 0.20 & 0.40 \\
MSb (II) & 5563 & 5.42 & 0.256 & -0.15 & 1.05 & -0.10 \\
MSb (III) & 5592 & 5.41 & 0.272 & -0.15 & 1.05 & -0.10 \\
\hline
\end{tabular}

Notes. For all the populations we assumed $[\mathrm{Fe} / \mathrm{H}]=-0.75$ and $[\alpha / \mathrm{Fe}]=0.4$.

choices for the abundances of $\mathrm{He}, \mathrm{C}, \mathrm{N}$, and $\mathrm{O}$. We will calculate synthetic colors for MSa and MSb stars for each of these options, seeking the composition that best reproduces all the observed color differences between the two MSs. All three of the options use the same abundance mixture for MSa. For He in MSa we choose the primordial He abundance, $Y=0.256$, and, following Cannon et al. (1998), we choose $[\mathrm{C} / \mathrm{Fe}]=$ 0.06 and $[\mathrm{N} / \mathrm{Fe}]=0.20$, which are typical for $\mathrm{CN}$-weak stars. As for $[\mathrm{O} / \mathrm{Fe}]$, we take 0.40 , typical for first-generation stars (Carretta et al. 2009a, 2009b). For MSb stars we try three different options. In Option I we assume that helium is the only cause of the MS split and adopt $Y=0.28$. In Option II we keep helium the same in the two populations but instead change the light-element abundances, adopting the values listed in Table 2. Finally, in Option III we adjust both helium and the light elements, again as given in Table 2.

We chose to characterize the fiducial sequences by measuring the color difference between MSa and MSb at the reference magnitude of $m_{\mathrm{F} 814 \mathrm{~W}}=18.2$, that is, the magnitude level indicated by the horizontal lines in Figure 9. For an assumed distance modulus of $(m-M)_{\mathrm{F} 814 \mathrm{~W}}=13.41$ and a reddening of $E(B-V)=0.04$, this corresponds to an absolute magnitude of $M_{\mathrm{F} 814 \mathrm{~W}}=4.85$. The adopted distance modulus and reddening are those that provide the best fitting of the isochrones to the data in the $m_{\mathrm{F} 606 \mathrm{~W}}$ versus $m_{\mathrm{F} 606 \mathrm{~W}}-m_{\mathrm{F} 814 \mathrm{~W}}$ plane and are in agreement with those provided by Gratton et al. (2003) and Harris (1996, 2010 December update). To determine the absorption in the $\mathrm{F} 814 \mathrm{~W}$ band we used the relations given by Bedin et al. (2005). We assumed $[\mathrm{Fe} / \mathrm{H}]=-0.75$ and $[\alpha / \mathrm{Fe}]=0.4$, in agreement with the values found by Carretta et al. (2009b). To compare the observations against synthetic photometry, we adopted the BaSTI isochrones ${ }^{13}$ (Pietrinferni et al. 2004, 2006) specifically calculated for the populations listed in Table 2, then determined $T_{\text {eff }}$ and $\log g$ for MS stars at $M_{\mathrm{F} 814 \mathrm{~W}}=4.85$. These temperatures and gravities were then used to calculate model atmospheres with the ATLAS12 code (Kurucz 2005; Castelli 2005; Sbordone 2005; Sbordone et al. 2007), which allows us to use arbitrary chemical compositions. Spectral synthesis from $2000 \AA$ to $10000 \AA$ was then performed using the SYNTHE code (Kurucz 2005; Sbordone et al. 2007), and the resulting synthetic spectra were integrated over the transmission of each of our nine filters to produce the synthetic magnitudes and colors.

We did this separately for an MSa star and for an $\mathrm{MSb}$ star, using for the latter each of the three composition options

\footnotetext{
13 http://www.oa-teramo.inaf.it/BASTI
} 

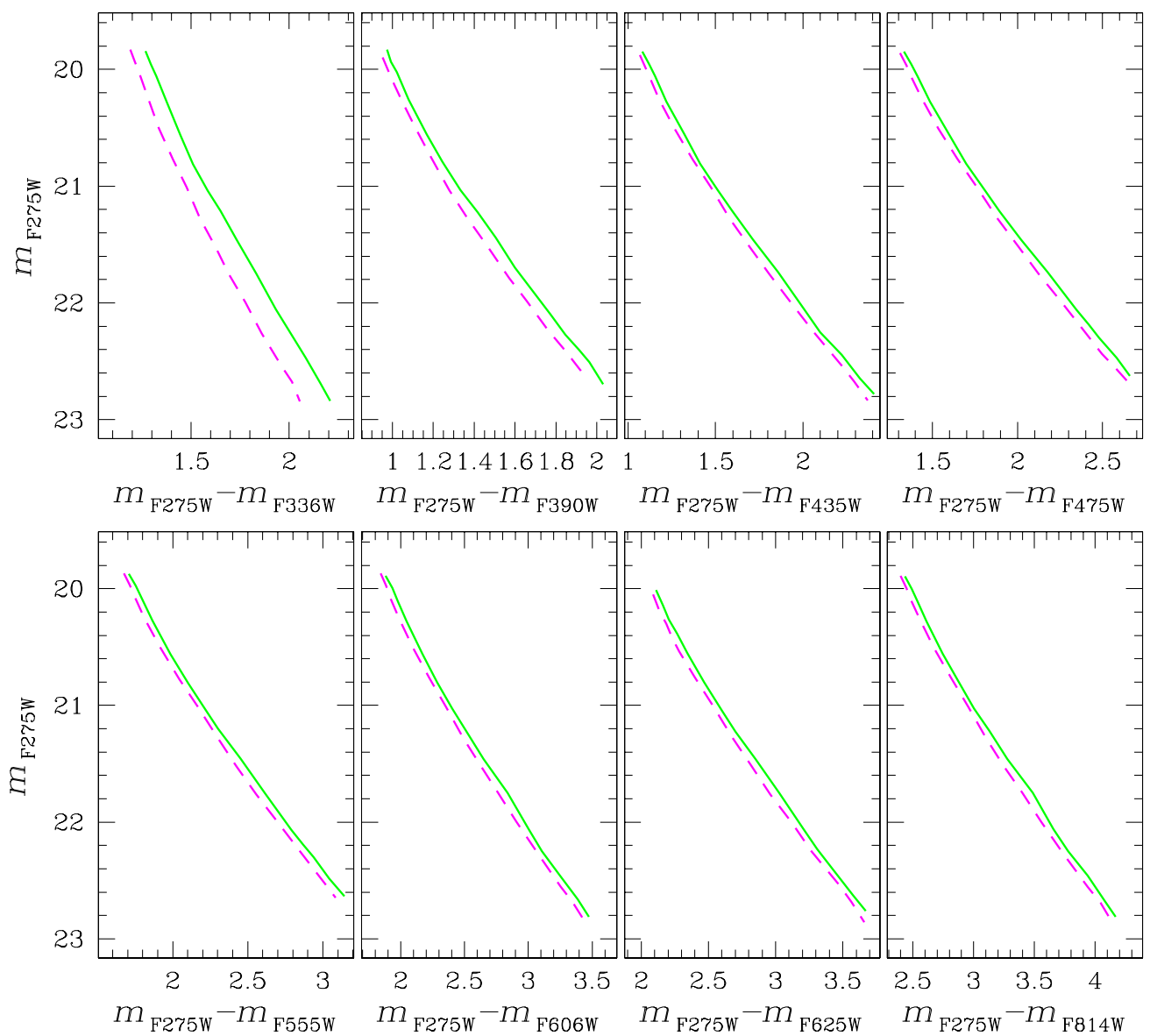

Figure 7. MS fiducial lines for MSa (green continuous line) and MSb (magenta dashed line) in the $m_{\mathrm{F} 275 \mathrm{~W}}$ vs. $m_{\mathrm{F} 275 \mathrm{~W}}-m_{\mathrm{X}}$ plane. In all these combinations of magnitude and colors the MSa stars are systematically redder than the MSb stars.

(A color version of this figure is available in the online journal.)

that Table 2 lists for MSb. We concentrate here on the eight $m_{\mathrm{X}}-m_{\mathrm{F} 814 \mathrm{~W}}$ colors, comparing the synthetic $\mathrm{MSa}-\mathrm{MSb}$ color differences with the observed ones shown in Figure 10. The results of our synthetic-spectrum calculations for Option I are shown in the figure as blue squares. At wavelengths longer than $\sim 4300 \AA$ there is good agreement with the observed color differences between the two MSs, but sizable discrepancies appear at shorter wavelengths, in particular in the F336W band, and we conclude that helium alone cannot account for the observed MS split. The colors that result from Option II (same helium but different CNO proportions) are plotted as gray triangles in Figure 10. Again there is a strong discrepancy between the simulated and the observed color differences in the two near-UV bands (F336W and F390W), but now in the opposite direction. Turning instead to Option III, with differences in both helium and the CNO elements, we see that the red asterisks in Figure 10 are in fair agreement with all of the observed color differences. The agreement is somewhat poorer for the F275W filter, but note that we have not fine-tuned the composition differences between the two MSs, and that UV colors can be very sensitive to the adopted mix of He and the CNO elements.

The effect of chemical composition differences on MS colors can be summarized as follows. The main effect of increased helium is to increase the temperatures, making all colors bluer. The effects of CNO are more subtle, since a specific molecule affects some bands but not others. Thus nitrogen affects the near-UV F336W and F390W bands via the NH band and CN bands, respectively, whereas oxygen affects the F275W band via the $\mathrm{OH}$ band. This is illustrated in the top panel of Figure 11, showing the synthetic spectra of an MSa and an MSb star as calculated for Option III (in green and magenta, respectively). The difference between the two is shown in the middle panel, while the bandpasses of our filters are plotted in the bottom panel.

These results are fully consistent with those obtained by Sbordone et al. (2011) and indicate that the observed color differences between $\mathrm{MSa}$ and $\mathrm{MSb}$ can be understood by assuming that MSa corresponds to a first stellar generation, with primordial $\mathrm{He}$, and O-rich/N-poor stars, whereas MSb corresponds to a population that is enriched in $\mathrm{He}$ and $\mathrm{N}$ but depleted in $\mathrm{O}$. This need for differences in both helium and $\mathrm{CNO}$ to account for all the color differences fits quite well with nucleosynthesis expectations, as helium-enriched stellar regions are also inevitably oxygen-depleted and nitrogenenriched, and vice versa-for example, in the layers subject to the second dredge-up, at the beginning of the asymptotic giant branch phase, or those subject to the so-called envelope-burning process, later during this phase (Renzini \& Voli 1981; Ventura \& D'Antona 2009).

\section{MULTIPLE STELLAR POPULATIONS ALONG THE SUBGIANT BRANCH}

The first photometric evidence for multiple populations in 47 Tuc came from the discovery of its bimodal SGB (Anderson 

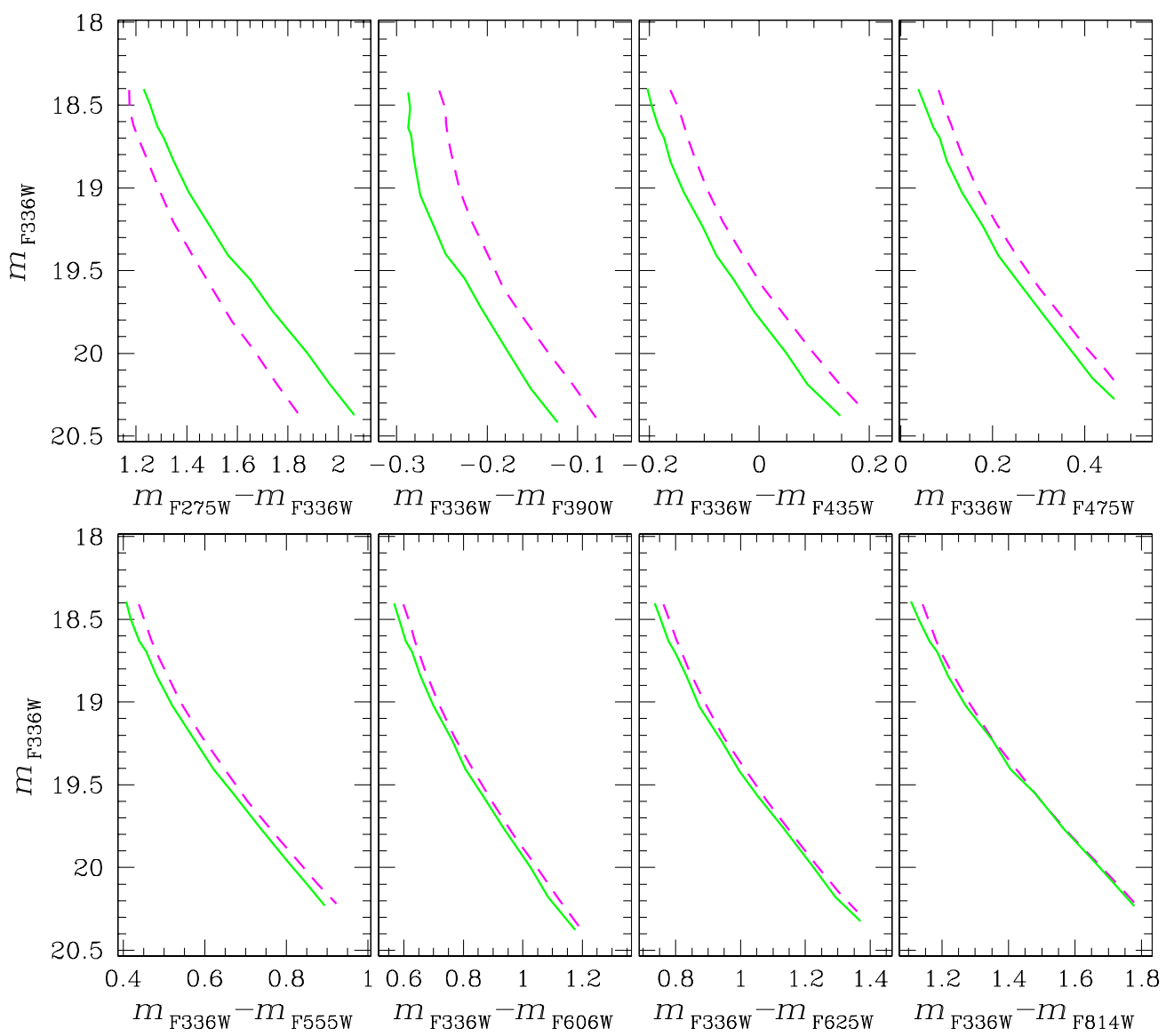

Figure 8. MS fiducial lines for MSa (green continuous line) and MSb (magenta dashed line) in the $m_{\mathrm{F} 336 \mathrm{~W}} \mathrm{vs} . m_{\mathrm{F} 336 \mathrm{~W}}-m_{\mathrm{X}}\left(m_{\mathrm{F} 275 \mathrm{~W}}-m_{\mathrm{F} 336 \mathrm{~W}}\right.$ in the upper left panel) plane. As in all the CMDs of Figure 7, MSb is bluer than MSa in all the $m_{\mathrm{F} 336 \mathrm{~W}}$ vs. $m_{\mathrm{F} 275 \mathrm{~W}}-m_{\mathrm{F} 336 \mathrm{~W}}$ CMDs. But notably, MSa becomes bluer than MSb in the $m_{\mathrm{F} 275 \mathrm{~W}}-m_{\mathrm{F} 336 \mathrm{~W}}$ color.

(A color version of this figure is available in the online journal.)

et al. 2009). These authors analyzed the large number of ACS/WFC archival HST images of the core of 47 Tuc and found that the SGB is split into two distinct components: a brighter SGB showing an intrinsic broadening in the F475W magnitude and a second one about 0.05 mag fainter, containing a minor fraction (a few per cent) of the stars. Anderson et al. also examined the SGB in an outer field, $\sim 6$ arcmin west of the cluster center, finding a similar vertical spread of the SGB. These SGBs have been further investigated by G. Piotto et al. (2011, in preparation), with a multi-band photometric analysis, showing that the magnitude difference between the faint and bright SGBs is almost constant in each of the nine HST filters they used, and finding a similar behavior in five other Galactic GCs that have multiple SGBs. Piotto et al. also found that the faint SGB makes up $8 \% \pm 3 \%$ of the stars in the central field of 47 Tuc. Di Criscienzo et al. (2010) suggest that the spread in luminosity of the bright SGB can be accounted for by a small spread in helium $(\Delta Y \sim 0.02)$, whereas they suggest that the minor population that makes up the faint SGB (fSGB) would be characterized by a small increase in $\mathrm{C}+\mathrm{N}+\mathrm{O}$.

In our multicolor set of CMDs, the SGB region turns out to be unexpectedly complex. Figure 12 shows in the left panel the visible-light CMD of $m_{\mathrm{F} 435 \mathrm{~W}}$ versus $m_{\mathrm{F} 606 \mathrm{~W}}-m_{\mathrm{F} 814 \mathrm{~W}}$, in which the fSGB component of Anderson et al., marked in red, is clearly visible as a separate sequence. In the right panels are the ultraviolet CMDs, with $m_{\mathrm{F} 275 \mathrm{~W}}$ versus $m_{\mathrm{F} 275 \mathrm{~W}}-m_{\mathrm{F} 336 \mathrm{~W}}$ and $m_{\mathrm{F} 275 \mathrm{~W}}$ versus $m_{\mathrm{F} 336 \mathrm{~W}}-m_{\mathrm{F} 435 \mathrm{~W}}$; in them the same stars instead fall on top of the numerically dominant sequence, which in turn splits into two separate sequences, bringing the total to three recognizable SGBs. How can this be?

Analogous to what we did for the MS stars, in Figure 13 we plot the UV two-color diagram of the SGB stars. Here again we see a bimodal distribution, and we therefore repeat the points on the right, and draw a separator line. Again by analogy, we color the stars on either side of the line green and magenta, respectively, and attach to the two regions the names SGBa and SGBb. In this case, however, we also distinguish with red X's the stars that belonged to the fSGB in the left panel of Figure 12.

The next step is to bring to bear on the SGB puzzle our full multicolor resources. In Figure 14, we show a $4 \times 4$ array of CMDs, with a different magnitude in each row, plotted against a different color in each column. Although these CMDs are worth looking at one by one, taken together they add up to an embarras de richesse. We therefore note for the reader the characteristics that strike us as systematic and significant.

1. SGBa stars share some similarities with MSa: in the CMDs that use the $m_{\mathrm{F} 275 \mathrm{~W}}-m_{\mathrm{F} 336 \mathrm{~W}}$ color they are on average redder than the other SGB stars, but when the $m_{\mathrm{F} 336 \mathrm{~W}}-m_{\mathrm{F} 435 \mathrm{~W}}$ color is used instead, the SGBa stars are on average bluer than the bulk of SGB stars. No significant color difference between the two SGB groups is evident in the other color combinations.

2. SGBa stars are typically brighter than SGBb stars in $m_{\mathrm{F} 336 \mathrm{~W}}$ (note in particular the third row of panels). Their being 


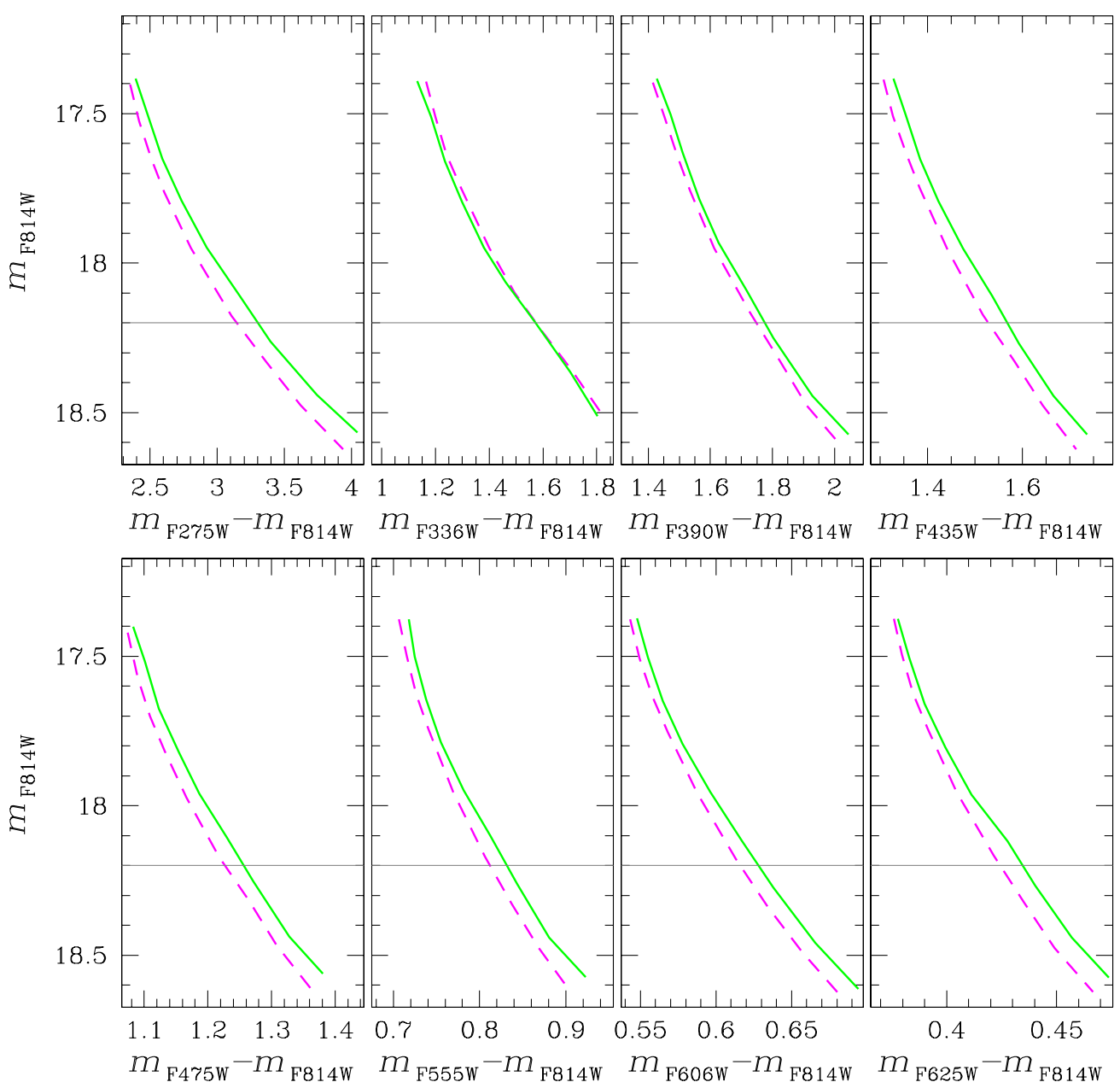

Figure 9. MS fiducial lines for MSa (green continuous line) and MSb (magenta dashed line) in the $m_{\mathrm{F} 814 \mathrm{~W}} \mathrm{vs.} m_{\mathrm{X}}-m_{\mathrm{F} 814 \mathrm{~W}}$ plane. In these combinations of magnitude and color the MSa stars are systematically redder than the MSb stars, apart from the $m_{\mathrm{F} 814 \mathrm{~W}}$ vs. $m_{\mathrm{F} 336 \mathrm{~W}}-m_{\mathrm{F} 814 \mathrm{~W}} \mathrm{CMD}$, where MSb is marginally redder than MSa. Horizontal gray lines mark the magnitude at which we have calculated the color difference between the two MSs $\left(m_{\mathrm{F} 814 \mathrm{~W}}=18.2\right.$; see the text for more details).

(A color version of this figure is available in the online journal.)

brighter than others in this band explains why they are redder than $\mathrm{SGBb}$ and $\mathrm{fSGB}$ in the $m_{\mathrm{F} 275 \mathrm{~W}}-m_{\mathrm{F} 336 \mathrm{~W}}$ color, and bluer than them in the $m_{\mathrm{F} 336 \mathrm{~W}}-m_{\mathrm{F} 435 \mathrm{~W}}$ color. No major systematic magnitude difference between SGBa and the two other SGB groups is apparent in any of the other panels.

3. In all of the CMDs the fSGB stars follow the same trend as the bulk of the SGBb stars, with the fSGB running parallel to $\mathrm{SGBb}$.

The nature of two of the three SGBs is now clear. In the first and second CMDs in the bottom row of Figure $14\left(m_{\mathrm{F} 275 \mathrm{~W}}\right.$ versus $m_{\mathrm{F} 275 \mathrm{~W}}-m_{\mathrm{F} 336 \mathrm{~W}}$ and $m_{\mathrm{F} 275 \mathrm{~W}}$ versus $\left.m_{\mathrm{F} 336 \mathrm{~W}}-m_{\mathrm{F} 435 \mathrm{~W}}\right)$ the green and magenta sequences interchange their order, exactly as we saw for MSa and MSb in these same colors (upper left and upper right CMDs in Figure 6). This justifies the name that we gave this pair of sequences, SGBa and SGBb, since they appear to be the direct descendants of MSa and MSb.

The sequence that we have marked in red establishes the existence of a third population in 47 Tuc, but we see it only here on the SGB; in other parts of the CMD we find no evidence of this third sequence. It is ironic that the very stars whose existence led us to study multiple populations in 47 Tuc should be left as an anomaly that does not fit into the picture that will emerge at the end of the present paper.

\section{MULTIPLE STELLAR POPULATIONS ON THE RED GIANT BRANCH}

Figure 15 shows the red giant region of 47 Tuc in three different CMDs. Both the $m_{\mathrm{F} 275 \mathrm{~W}}$ versus $m_{\mathrm{F} 275 \mathrm{~W}}-m_{\mathrm{F} 336 \mathrm{~W}}$ and the $m_{\mathrm{F} 336 \mathrm{~W}}$ versus $m_{\mathrm{F} 336 \mathrm{~W}}-m_{\mathrm{F} 435 \mathrm{~W}}$ diagrams suggest that the RGB is intrinsically broad; the observed color spread of $0.1-0.2$ mag is much larger than the photometric error, which for these bright RGB stars is less than 0.02 mag in color, and there is even some hint of a double color distribution. However, in the $m_{\mathrm{F} 435 \mathrm{~W}}$ versus $m_{\mathrm{F} 435 \mathrm{~W}}-m_{\mathrm{F} 814 \mathrm{~W}} \mathrm{CMD}$ (rightmost panel) the RGB is quite narrow.

To investigate this behavior further, we again turn to the $m_{\mathrm{F} 275 \mathrm{~W}}-m_{\mathrm{F} 336 \mathrm{~W}}$ versus $m_{\mathrm{F} 336 \mathrm{~W}}-m_{\mathrm{F} 435 \mathrm{~W}}$ two-color diagram. In panel (a) of Figure 16, we show a zoomed CMD, in which the RGB stars are indicated in black. Panel (b) shows the twocolor diagram of those stars. The distribution of stars in the diagram is clearly bimodal, and in panel (c) we arbitrarily draw a line separating two groups of stars, which we name RGBa and $\mathrm{RGBb}$, again color-coded green and magenta, respectively. The lower panels of the figure show the steps by which numerical fractions are derived for the RGBa and RGBb populations. In accordance with the procedure introduced in Section 3, we derived a fiducial line for RGBb in the two-color diagram; the line is shown in panel (d). In panel (e), we have subtracted 


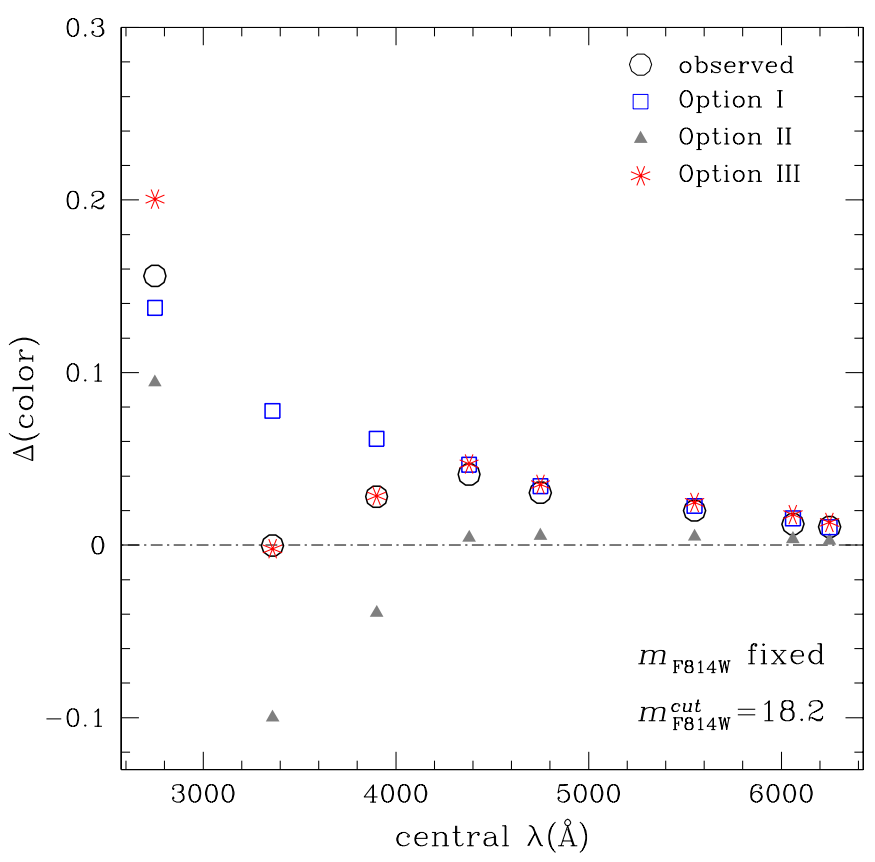

Figure 10. Color separations of the ridgelines of MSa and MSb for differen color baselines. The colors used are $m_{\mathrm{X}}-m_{\mathrm{F} 814 \mathrm{~W}}$, where $\mathrm{X}$ is one of the other eight filters. Each color separation is shown at the central wavelength of its filter X. (For example, the leftmost point is the separation of the two sequences in $m_{\mathrm{F} 275 \mathrm{~W}}-m_{\mathrm{F} 814 \mathrm{~W}}$.) All color separations are measured at $m_{\mathrm{F} 814 \mathrm{~W}}=18.2$. A positive value of $\Delta$ (color) means that MSa is bluer; negative would mean that $\mathrm{MSa}$ is redder. Observations are plotted as open circles, while the color differences expected from theoretical Options I, II, and III are shown as blue squares, gray triangles, and red asterisks, respectively.

(A color version of this figure is available in the online journal.)

from the color of each star the color of the fiducial line at the magnitude of the star, so as to verticalize the sequences. In panel (f), we plot a histogram of the verticalized colors and fit it with Gaussians to represent the RGBa and RGBb populations. The result is that $\mathrm{RGBa}$ represents $19 \% \pm 3 \%$ of the total.

\subsection{The Double RGB in the Outer Parts of the Cluster}

Ground-based photometry available for a field of view that goes out to 25 arcmin allows us to investigate the radial behavior of the populations. However, the lack of an equivalent to the F275W passband deprives us of our sharpest tool, and we must look for the best population discriminant among the groundbased passbands that are available. Fortunately, ground-based $U, B, V$, and $I$ are quite similar to our HST F336W, F435W, F555W, and F814W bands. Our procedure, then, was as follows. Since our use of the F275W and F336W bands had separated the RGBa and RGBb stars, we took those two samples of stars and examined their behavior in the many different diagrams that can be plotted using only the four bands that have ground-based equivalents, in order to see if we could find a surrogate for the two missing bands. As a result, we found that RGB stars separate into two groups when we plot $m_{\mathrm{F} 435 \mathrm{~W}}$ against the combination $m_{\mathrm{F} 336 \mathrm{~W}}+m_{\mathrm{F} 814 \mathrm{~W}}-m_{\mathrm{F} 435 \mathrm{~W}}$, as is shown in Figure 17.

Just to demonstrate the effectiveness of this separation, we use it to separate the stars of RGBa and RGBb, instead of using the easier route offered by Figure 16. As in similar situations encountered earlier, we draw a fiducial line for RGBb, by putting a spline through the median colors in successive short intervals of magnitude, and doing an iterated sigma clipping of outliers. We again subtract the color of the fiducial line from the color

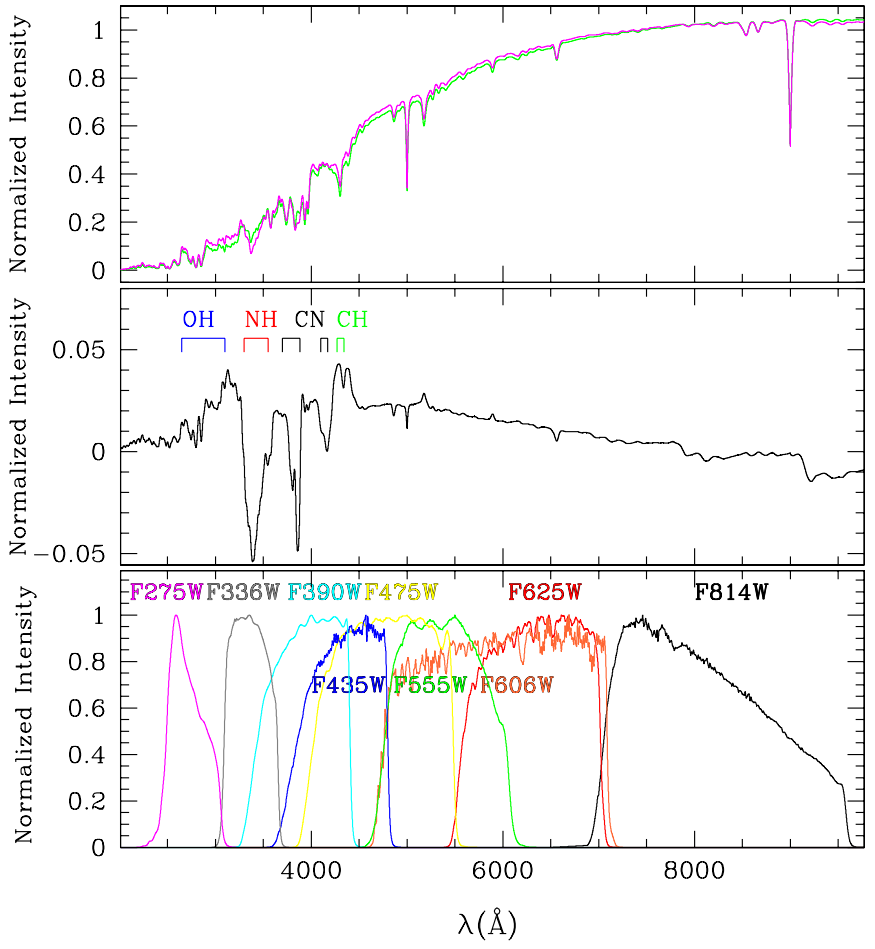

Figure 11. Upper panel: comparison of the synthetic spectra of an MSa star (green) and an MSb star (magenta). See the text for more details. Middle panel: difference between the spectra of the MSa star and the MSb star. Lower panel: normalized responses of the HST filters used in this paper.

(A color version of this figure is available in the online journal.)

of each star to produce the verticalized colors shown in the left panel of Figure 18. The corresponding two-Gaussian fit to the color distributions of the resulting $\mathrm{RGBa}$ and $\mathrm{RGBb}$ stars is shown in the right panel of the figure.

Finally, in Figure 19, which is analogous to Figure 16, we use $U, B$, and $I$ magnitudes to separate RGBa and RGBb stars in our entire ground-based field, in exactly the same way as we demonstrated with our HST photometry using the corresponding F336W, F435W, and F814W passbands. Then we calculate the difference between the $U+I-B$ value of each star and the value of the fiducial line at the magnitude of the star, $\Delta(U+I-B)$. This process makes the RGB vertical, and we show that in the left panel of Figure 20. The corresponding histograms are plotted in the right panel of the figure, again fitted by two overlapping Gaussians.

Having developed a method of using ground-based filters for separating populations, we now study the spatial distribution of the two RGB components by dividing our ground-based field of view into three annuli, each containing about the same number of RGB stars, and applying the procedure described above to each group separately. The histograms of the $\Delta(U+I-B)$ distributions are shown in Figure 21. It is clear from this figure that $\mathrm{RGBb}$ is more centrally concentrated than RGBa, with the fraction of RGBa stars ranging from $0.22 \pm 0.04$ in the $1.7-3.5$ arcmin bin, to $0.32 \pm 0.04$ at radial distances from 3.5 to 7.8 arcmin, up to $0.43 \pm 0.04$ for stars in the $7.8-25$ arcmin bin. This result will be discussed in detail in Section 8 , where we also compare the radial distributions of the two RGB components with those of MS and HB components.

We finally note that we carefully analyzed the SGB and the MS in the $B$ versus $U+I-B$ diagram, the same as we have just done for the RGB, but for those other regions we found 

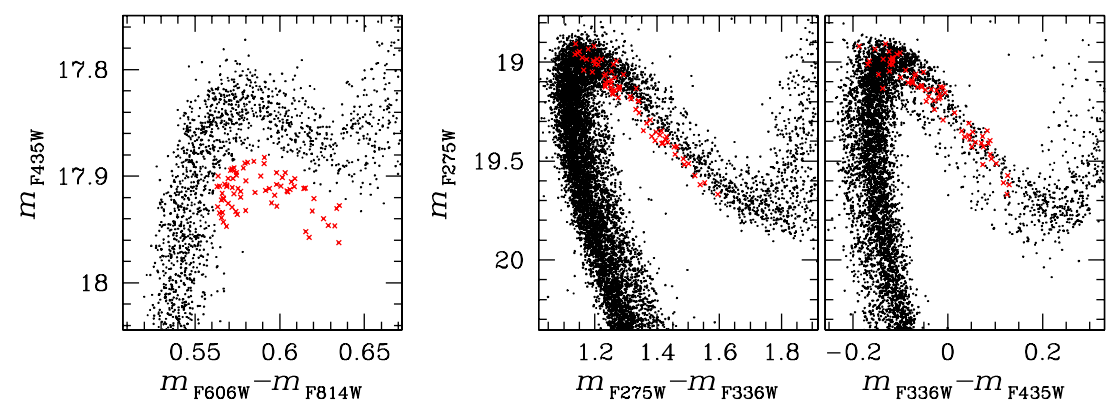

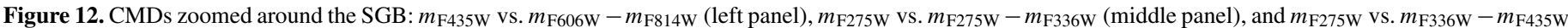
(right panel). The leftmost CMD has been used to select a sample of faint SGB stars, represented by red X's in all three CMDs.

(A color version of this figure is available in the online journal.)

no evidence for multiple sequences, presumably because of the larger errors in the ground-based photometry of those fainter stars.

\subsection{The Chemical Content of Stars in the Two RGB Sequences}

Since the red giants are the brightest stars in 47 Tuc, their spectroscopy has a long history. In the early seventies, large star-to-star cyanogen variations showed that the cluster is not chemically homogeneous (e.g., McClure \& Osborn 1974; Bell et al. 1975; Hesser et al. 1977; Norris 1978; Hesser 1978), and in a paper that sets a standard of spectroscopic accuracy at that time, Norris \& Freeman (1979) measured CN in 142 RGB stars of 47 Tuc and found a bimodal distribution, with $\mathrm{CN}$-strong stars more centrally concentrated. Our Figure 22 is adapted from their Figure 1, and here we plot their values of the David Dunlap Observatory $C(4142)$ index (which is sensitive to the blue $\mathrm{CN}$ bands) against our $V$ magnitude. The dashed line separates the $\mathrm{CN}$-strong and $\mathrm{CN}$-weak stars, which for added emphasis we have indicated by magenta circles and green triangles, respectively. We have 80 RGB stars in common with Norris \& Freeman, and in Figure 23 we have marked their $\mathrm{CN}$-strong and $\mathrm{CN}$-weak stars with distinctive symbols as defined above, in a repeat of our Figure 19. Note that most of the $\mathrm{CN}$-strong stars belong to $\mathrm{RGBb}$, and nearly all the $\mathrm{CN}$-weak stars lie on RGBa. It is obviously very tempting to associate the two groups of $\mathrm{CN}$-weak and $\mathrm{CN}$-strong stars with the two RGBs. More accurate measurements would be needed, however, to establish whether the few exceptions to this rule are real or are due to errors in either the photometric or the spectroscopic measures.

It is now well established that a chemical signature of multiple stellar populations in GCs is offered by the $\mathrm{Na}-\mathrm{O}$ anticorrelation that has been noticed in virtually every cluster observed so far. The Na-O anticorrelation in RGB stars of 47 Tuc has recently been studied by Carretta et al. (2009b), and their data are shown in Figure 24, where we have made an arbitrary division into Na-poor/O-rich stars (green triangles) and Na-rich/O-poor stars (magenta circles). The $U$ versus $U-B$ CMD shown in Figure 25 gives photometric evidence for a spread in color that extends from the base of the RGB to its tip. We then identify stars from Carretta et al. (2009b) in our ground-based CMDs of 47 Tuc, and the two groups of stars defined in Figure 24 are found to segregate on the RGB as illustrated in Figure 25, with Na-rich/O-poor stars being systematically bluer in $U-B$ compared to the Na-poor/O-rich stars, while mixing with them in the $B$ versus $B-I C M D$, in analogy with the results of Marino et al. (2008) for M4.

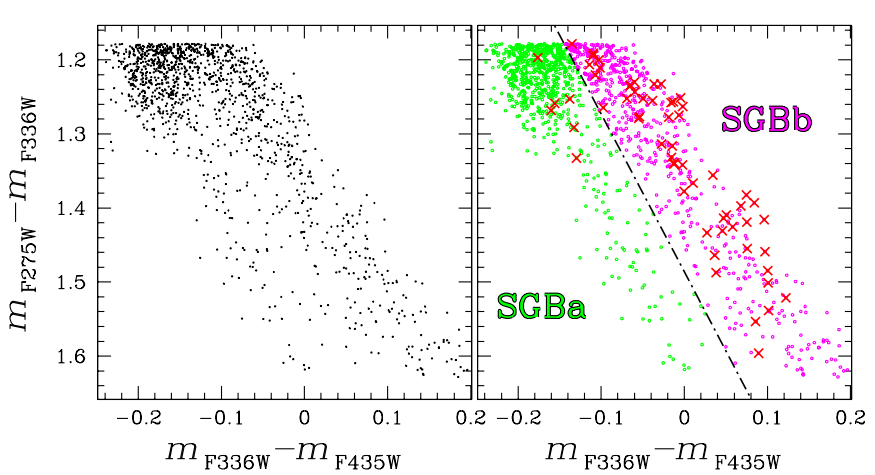

Figure 13. $m_{\mathrm{F} 275 \mathrm{~W}}-m_{\mathrm{F} 336 \mathrm{~W}}$ vs. $m_{\mathrm{F} 336 \mathrm{~W}}-m_{\mathrm{F} 435 \mathrm{~W}}$ two-color diagram for $\mathrm{SGB}$ stars. The dot-dashed line in the right panel arbitrarily separates the SGBa and $\mathrm{SGBb}$ stars, which are plotted green and magenta, respectively.

(A color version of this figure is available in the online journal.)

Finally, in Figure 26 we plot the Na-poor/O-rich and Narich/O-poor stars in the $B$ versus $U+I-B$ diagram and in the $B$ versus $\Delta(U+I-B)$ diagram to investigate the abundances of $\mathrm{Na}$ and $\mathrm{O}$ in the two RGBs. We find that RGBa is populated mainly by Na-poor/O-rich stars, while all the Na-rich/O-poor stars belong to RGBb.

\section{MULTIPLE STELLAR POPULATIONS ON THE HORIZONTAL BRANCH}

We now turn to the HB of 47 Tuc. Figure 27 shows the HST data in the same plots as were used for the other sequences. In order to point out the stars that we are studying here, we show in panel (a) a long-wavelength CMD, with the HB emphasized in black. In panel (b) we show the ultraviolet two-color diagram of these stars, with the two HB sequences $\mathrm{HBa}$ and $\mathrm{HBb}$ identified as usual in panel (c). In panel (d) we show the same plot again, but with a fiducial line through the HBb locus. We verticalize the sequences in the usual way in panel (e). Panel (f) shows the corresponding histogram, with the fit by two Gaussians that are colored appropriately. Perhaps quite unsurprisingly at this stage, the result that HBa makes up $19 \% \pm 3 \%$ of the HB stars is very similar to what we found for the RGB.

To study the radial distribution of the two HB populations, we again use ground-based photometry. In panel (a) of Figure 28, we show the $B$ versus $B-I C M D$ of all the stars that passed the selection criteria described in Section 2, and the selected HB stars are marked in black. Panel (b) shows a zoom of the HB, in the same color system, and in panel (c) we plot the same stars in the $B$ versus $U+I-B$ diagram, where the distribution shows some bimodality. The red dashed line is the fiducial line of the 


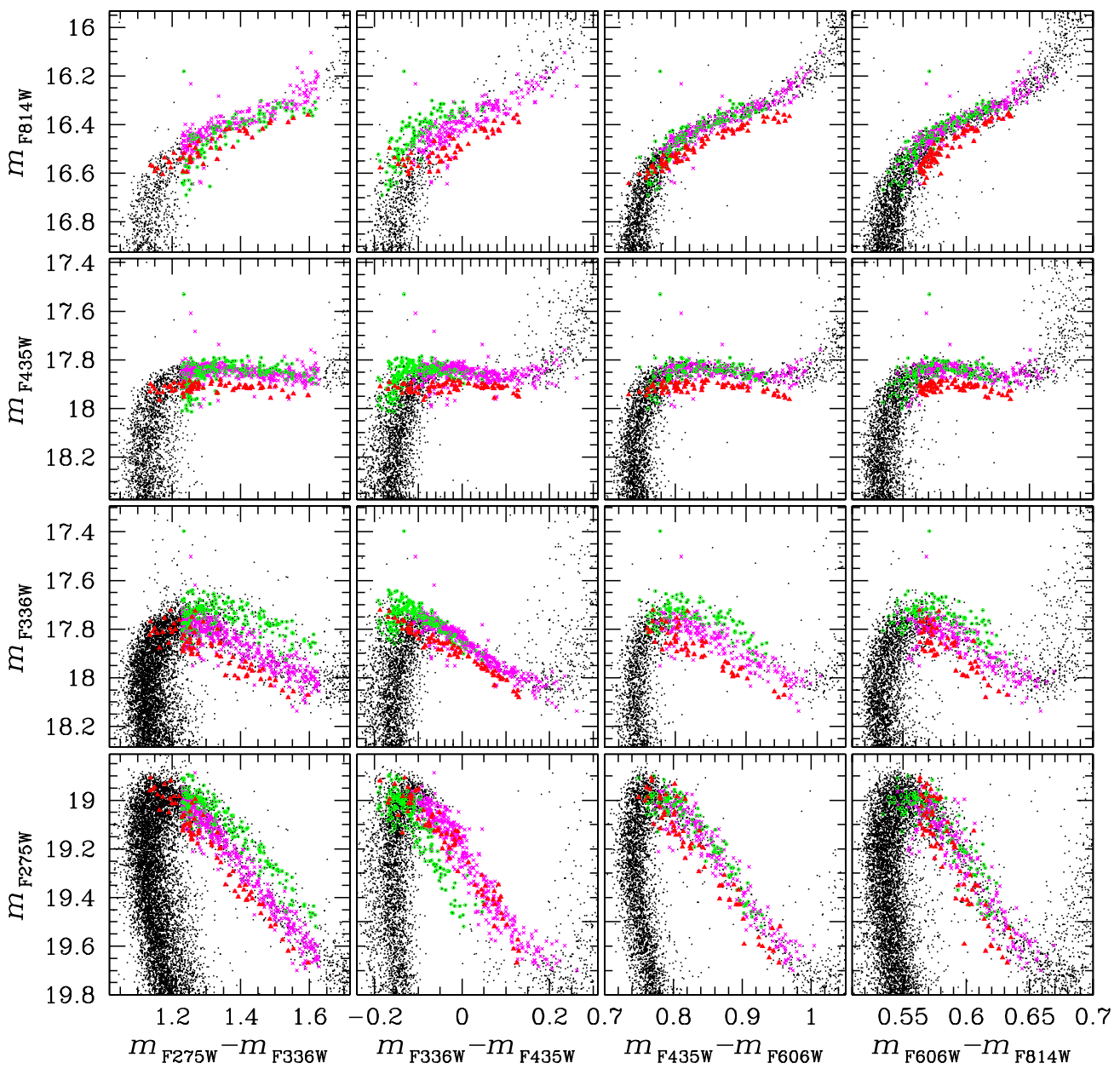

Figure 14. Collection of CMDs from HST photometry, zoomed around the SGB. The two groups of SGBa and SGBb stars defined in Figure 13 are plotted in green and magenta, respectively, while we have used red symbols for the faint SGB stars selected in Figure 12.

(A color version of this figure is available in the online journal.)
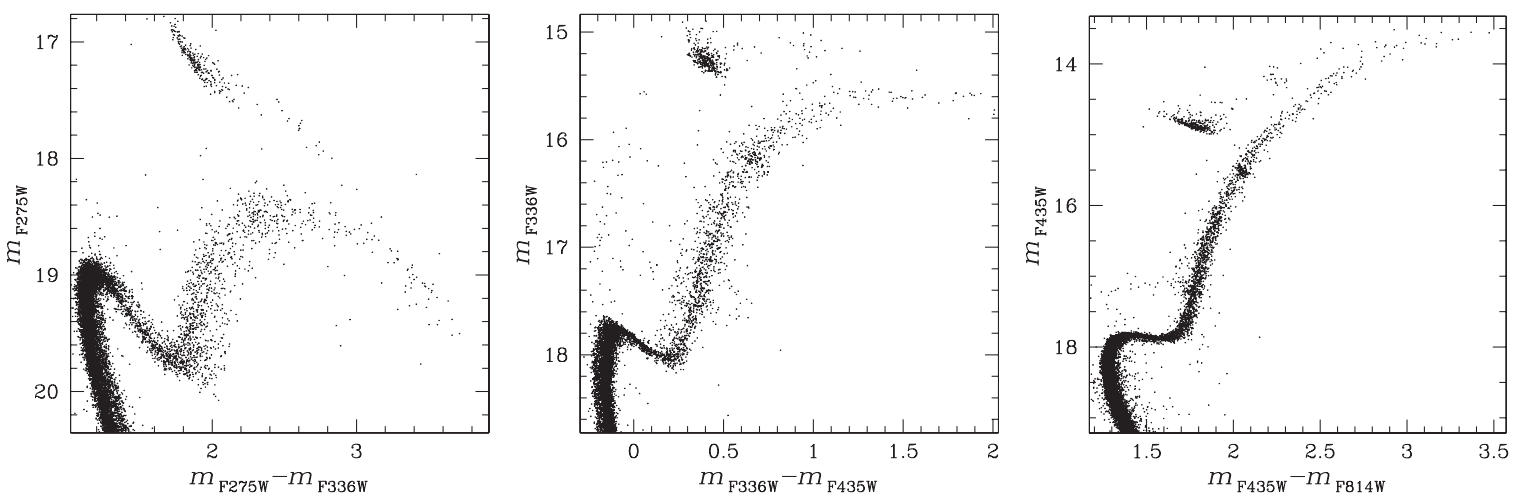

Figure 15. This figure illustrates the three CMDs from HST photometry that best summarize the behavior of RGB stars. Note the wide color spread of $\sim 0.1-0.2$ mag in the left and the middle CMDs, which also show some hints of color bimodality. The RGB becomes narrower and well defined in the right-hand CMD.

more populous $\mathrm{HB}$ component, and analogous to the previous figure we refer to the lower-left stars as $\mathrm{HBa}$ and the upper-right stars as $\mathrm{HBb}$. The rectified $B$ versus $U+I-B$ plot is shown in panel (d), and the corresponding histogram is shown in panel (e). In Figure 29 we compare the histogram distribution of HB stars with different radial distance from the cluster center.

It has been suspected for a long time that the HB of 47 Tuc might contain multiple populations. Norris \& Freeman (1982) measured the strengths of $\mathrm{CN}$ and $\mathrm{CH}$ bands in the spectra of $14 \mathrm{HB}$ stars and concluded that their results were "consistent with a dichotomy as found for the giants," which agrees with the result that we have just presented. They also noted that $\mathrm{CN}$-weak stars are on average $\sim 0.04$ mag brighter in $V$ than $\mathrm{CN}$-strong stars-which is similar to what we see in panel (e) of Figure 27 and panel (d) of Figure 28.

Figure 1(a) from Norris \& Freeman (1982) is reproduced here as Figure 30. We plotted their $S(3839)$ index measurements for $14 \mathrm{HB}$ stars as a function of the $(B-V)$ color and drew the line that shows the dependence of $S(3839)$ on effective temperature, as discussed by Norris \& Freeman (1982). The two groups of 

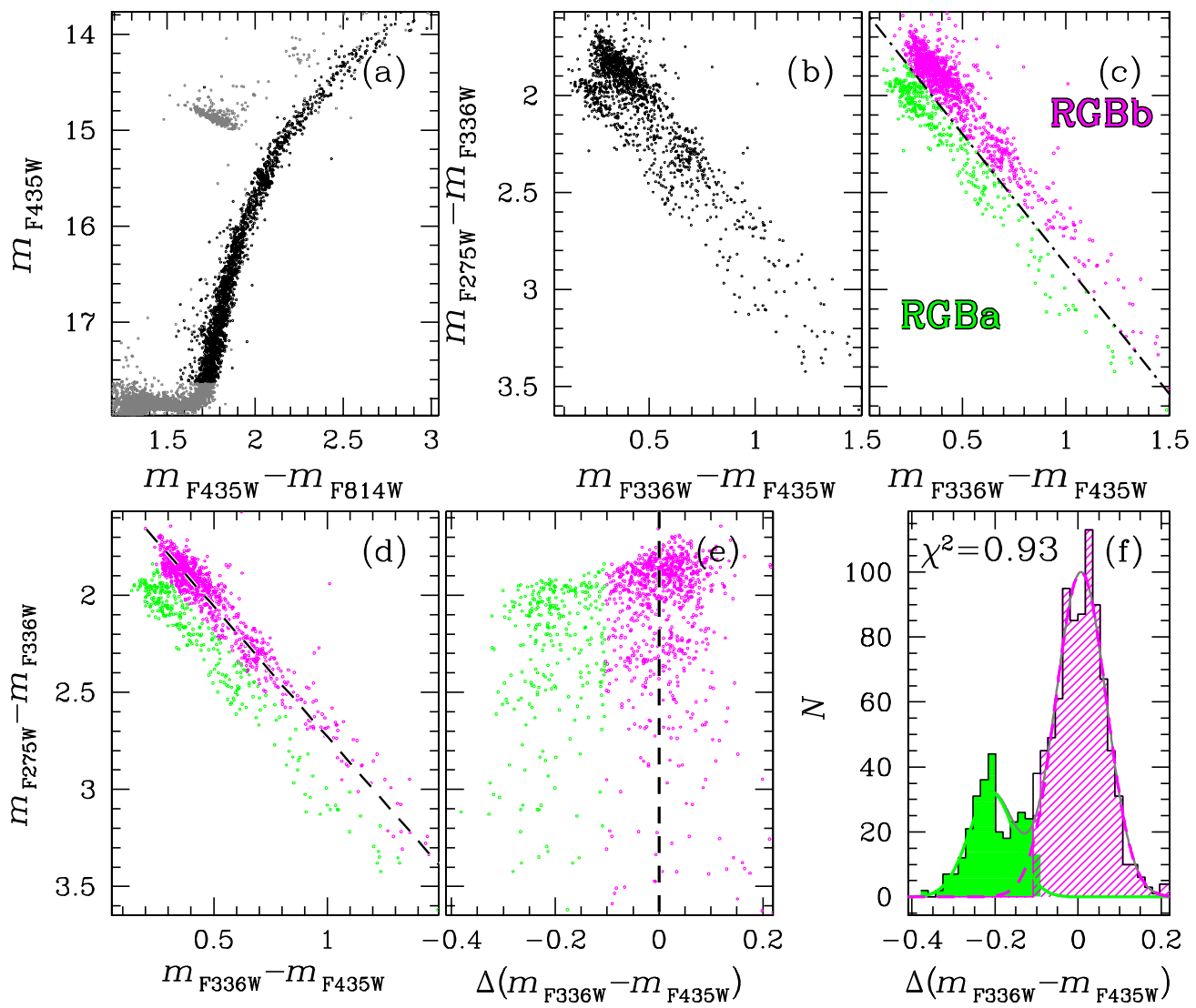

Figure 16. Panel (a): $m_{\mathrm{F} 435 \mathrm{~W}}$ vs. $m_{\mathrm{F} 435 \mathrm{~W}}-m_{\mathrm{F} 814 \mathrm{~W}} \mathrm{CMD}$ from ACS/WFC photometry. The black points are the RGB stars that we show in the other panels. (b) $m_{\mathrm{F} 275 \mathrm{~W}}-m_{\mathrm{F} 336 \mathrm{~W}}$ vs. $m_{\mathrm{F} 336 \mathrm{~W}}-m_{\mathrm{F} 435 \mathrm{~W}}$ two-color diagram for these stars. (c) We arbitrarily drew by hand the dash-dotted line that separates RGBa (green) and RGBb (magenta). (d) The dashed line is the fiducial sequence of the RGBb stars, drawn by the fitting method that we described in Section 3. (e) The verticalized colors of the stars. (f) Histogram of the colors in panel (e), with a fit by two Gaussians, whose sum is shown by the solid gray line.

(A color version of this figure is available in the online journal.)

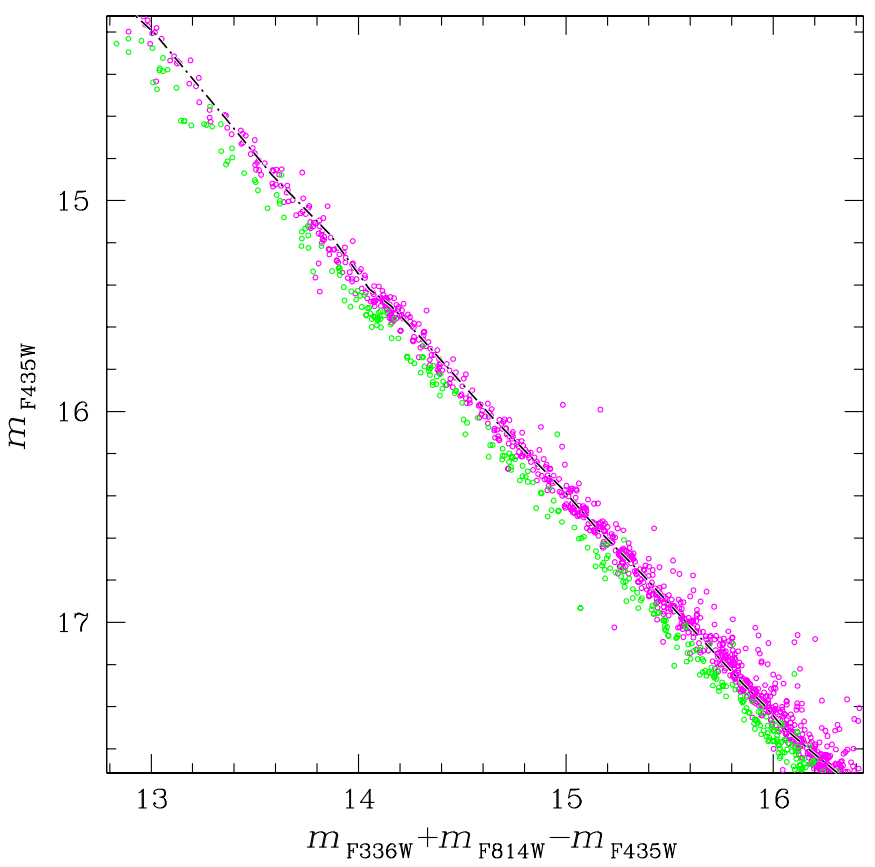

Figure 17. $m_{\mathrm{F} 435 \mathrm{~W}}$ vs. $m_{\mathrm{F} 336 \mathrm{~W}}+m_{\mathrm{F} 814 \mathrm{~W}}-m_{\mathrm{F} 435 \mathrm{~W}}$ diagram for RGB stars. The dash-dotted black line is the fiducial line of the RGBb sequence.

(A color version of this figure is available in the online journal.)
$\mathrm{CN}$-weak and $\mathrm{CN}$-strong stars defined by Norris \& Freeman are plotted as green triangles and magenta circles, respectively. Our ground-based catalog has in common 9 out these 14 stars. These stars are marked with black circles. Among them three are $\mathrm{CN}$-strong and six are $\mathrm{CN}$-weak stars.

In Figure 31, we show the position in the $B$ versus $B-I$ CMD (right panel) and in the $B$ versus $\Delta(U+I-B)$ diagram the nine stars in common (left panel). CN-strong stars are on average brighter than $\mathrm{CN}$-weak ones in the $B$ band. The right panel shows that $\mathrm{CN}$-strong and $\mathrm{CN}$-weak stars have $\Delta(U+I-B)$ values consistent with that of the bulk of the $\mathrm{HBb}$ and $\mathrm{HBa}$ groups, respectively. An exception to this rule is given by a $\mathrm{CN}$ weak star which has a $B$ magnitude and $\Delta(U+I-B)$ value close to that of $\mathrm{HBb}$ stars. It is not clear if this anomalous position in the latter diagram is an intrinsic property of this star or is due to either photometric or spectroscopic errors.

The connection between the HB morphology in GCs and the groups of stars with different abundances of light elements $(\mathrm{Na}, \mathrm{O}, \mathrm{C}, \mathrm{N}$ ) is not a peculiarity of 47 Tuc but has also been observed in the GC NGC 6121 (M4). As already mentioned in Section 1, this cluster hosts two groups of stars with different $\mathrm{Na}$ and $\mathrm{O}$ content (Marino et al. 2008), as well as a bimodal HB. Marino et al. (2011a) have recently measured oxygen and sodium for stars in the blue and the red HB segments of M4, and found that the red HB is made by stars with low $\mathrm{Na}$ and high $\mathrm{O}$ content, while blue HB stars are all Na-enhanced and O-depleted (possibly He-rich). 

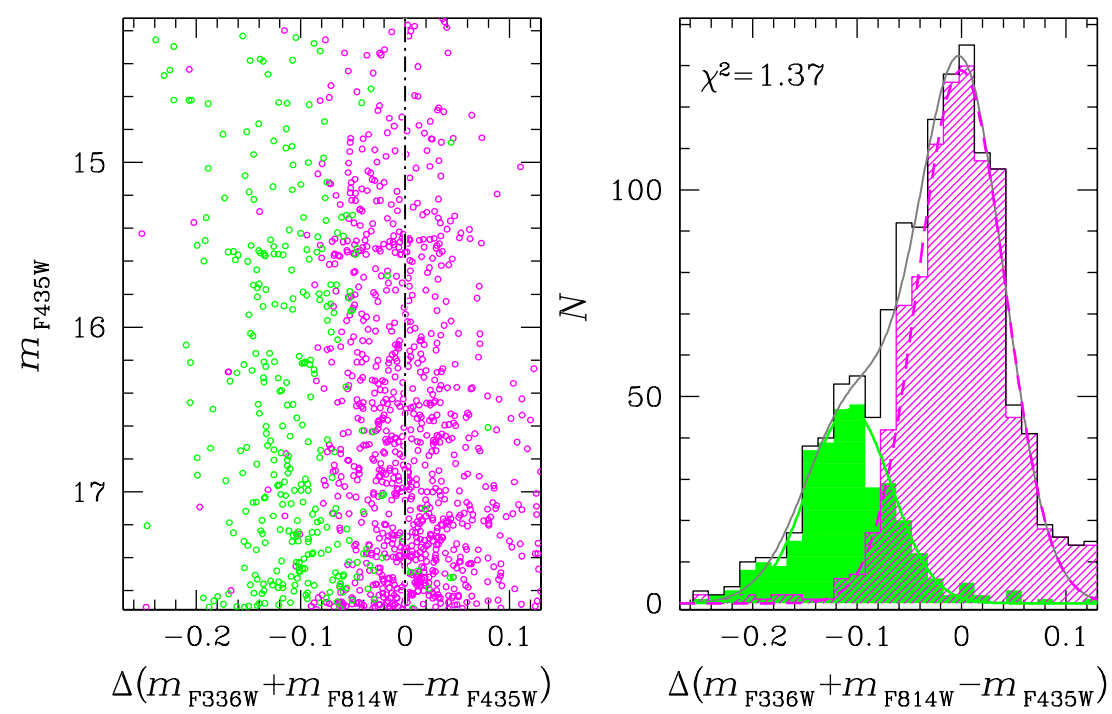

Figure 18. Left: the same points as in Figure 17, but after subtracting from the $m_{\mathrm{F} 336 \mathrm{~W}}+m_{\mathrm{F} 814 \mathrm{~W}}-m_{\mathrm{F} 435 \mathrm{~W}}$ value of each star the value of the fiducial at the same magnitude. Right: histogram of the colors in the left panel. The gray line is the best fit of two Gaussians, which are plotted as dotted and dashed black lines, and are shaded green and magenta, respectively.

(A color version of this figure is available in the online journal.)
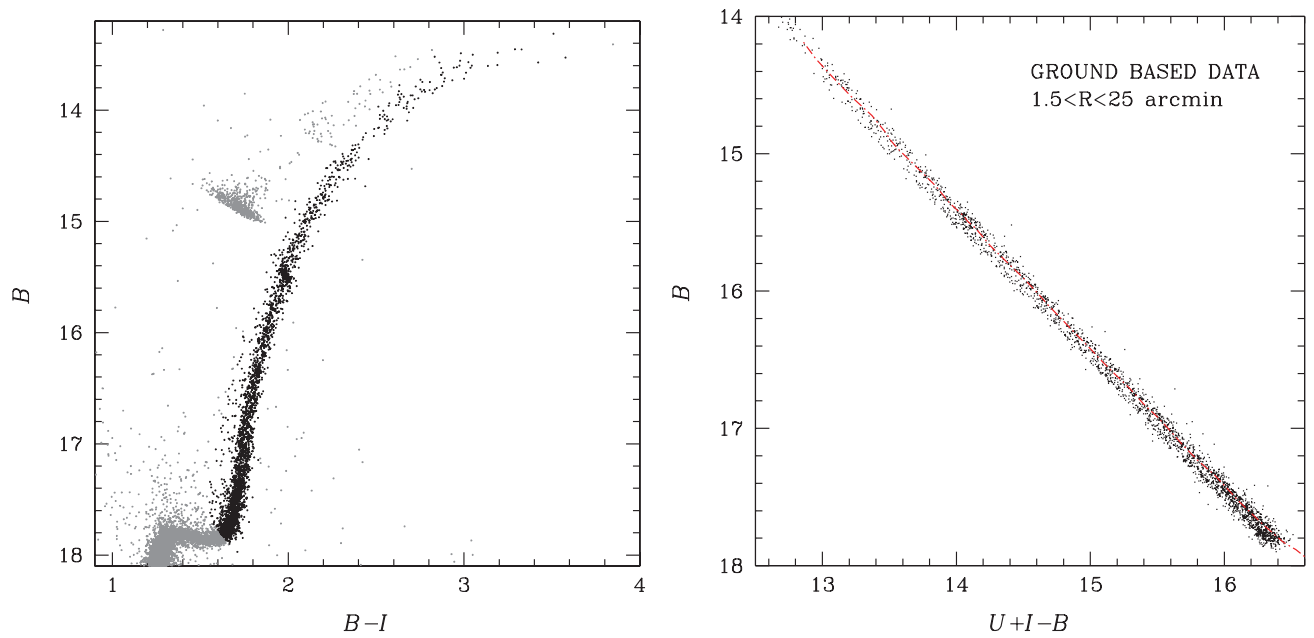

Figure 19. Similar to what was shown in Figure 17, but now using the ground-based photometry for the RGB stars. The left panel is a $(B, B-I)$ CMD in which the black points are the RGB stars that will be used for the separation of RGBa and RGBb. On the right is the CMD in $B$ vs. $U+I-B$, with a red fiducial line drawn through the RGBb stars.

(A color version of this figure is available in the online journal.)

These results on 47 Tuc and NGC 6121 provide direct evidence that the HB morphology of these GCs is strictly related to the multiple stellar generations they host, and suggest that the multiple sequences discovered in the CMDs of many GCs may be connected with the HB morphology.

\subsection{The Role of $C, N$, and $O$ on the $S G B$ and the $H B$}

In order to better understand the origin of the multicolor distribution of SGB and HB stars, we present here an analysis similar to that described in Section 4 for MS stars, using a number of synthetic spectra over the wavelength range of interest (i.e., $2000 \AA<\lambda<5000 \AA$ ). They were computed using the Kurucz (1993) model atmospheres (with the overshooting option switched off), and line lists from the Kurucz CD-ROMs. Although these lists may be incomplete, especially in the UV, and the models adopt the one-dimensional approximation, the resulting model atmospheres are still useful for the present pur- poses, which consist of identifying the major spectroscopic features that can affect the photometry of stars in 47 Tuc. In the rest of this discussion, we limit ourselves to consideration of differential effects between stars that should have very similar spectra unless their chemical compositions differ, a subgiant star with $T_{\text {eff }}=5700 \mathrm{~K}$ and $\log g=3.75$, and a red HB star with $T_{\text {eff }}=5400 \mathrm{~K}$ and $\log g=2.60$.

One set of synthetic spectra was computed with the typical abundance pattern observed in metal-poor stars in the field, $[\mathrm{C} / \mathrm{Fe}]=[\mathrm{N} / \mathrm{Fe}]=0.0,[\mathrm{O} / \mathrm{Fe}]=0.4$; spectra computed with these parameters are called "N-poor." They should mimic the composition of the stars that Carretta et al. (2009b) call primordial-generation. We computed another pair of spectra for the same atmospheric parameters, now with $[\mathrm{C} / \mathrm{Fe}]=-0.2$, $[\mathrm{N} / \mathrm{Fe}]=+1.3$, and $[\mathrm{O} / \mathrm{Fe}]=-0.1$, but with the other element abundances the same as in the "N-poor" stars. We call this second group "N-rich"; they should mimic the spectra of the stars that Carretta et al. (2009b) call second generation. For 

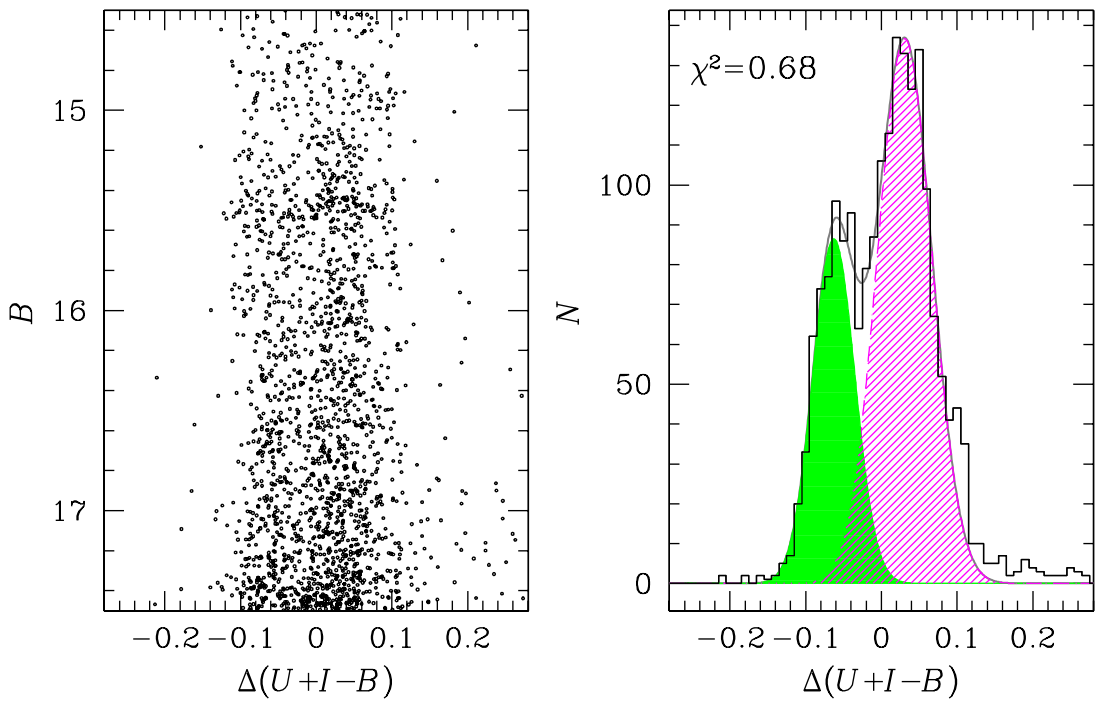

Figure 20. Left: same diagram as in Figure 19 but after subtracting the RGB fiducial. Right: histograms of $\Delta(U+I-B)$ for RGB stars in our ground-based photometry.

(A color version of this figure is available in the online journal.)

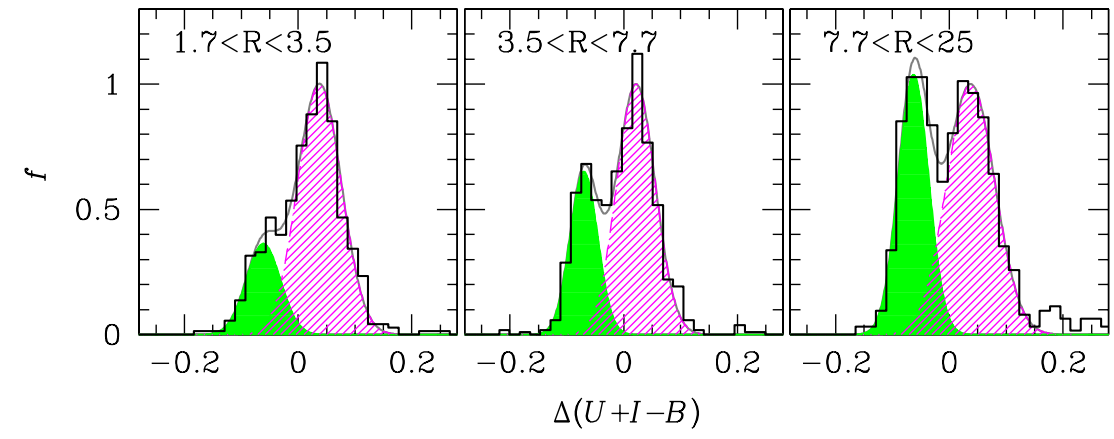

Figure 21. Histogram of the $\Delta(U+I-B)$ distributions of RGB stars at three different radial distances.

(A color version of this figure is available in the online journal.)

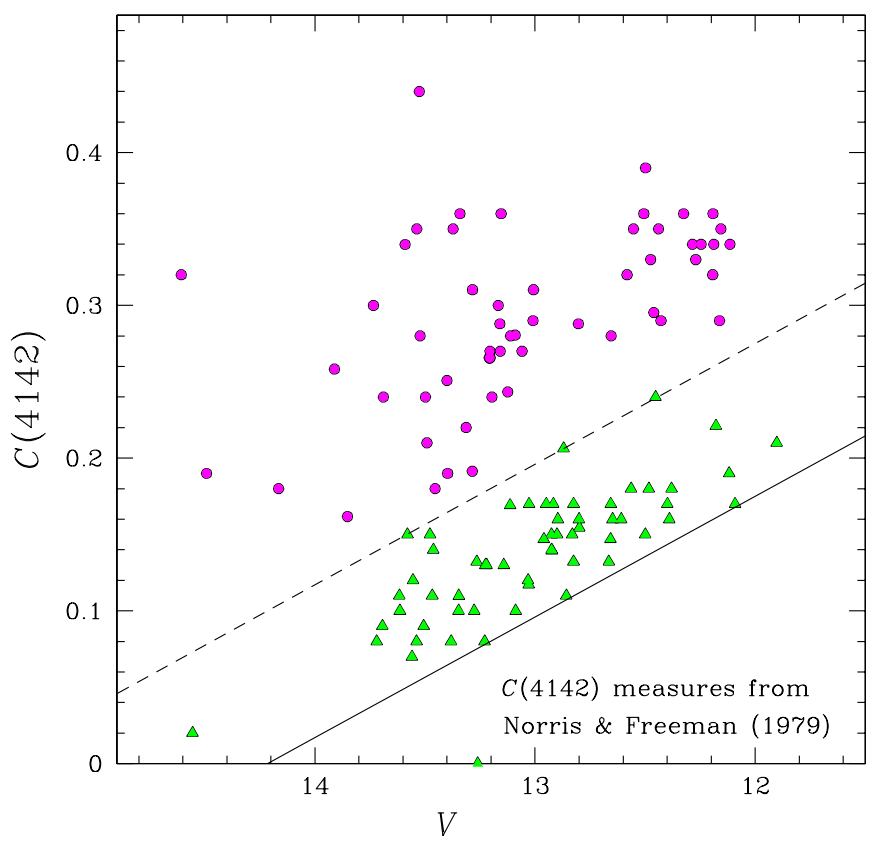

Figure 22. Adaptation of Figure 1 of Norris \& Freeman (1979), showing $C(4142)$ index vs. $V$ magnitude for their sample of RGB stars. Their full line follows the lower bound of the data, while our dashed line separates the $\mathrm{CN}$ weak stars (green triangles) from the $\mathrm{CN}$-strong ones (magenta circles).

(A color version of this figure is available in the online journal.)

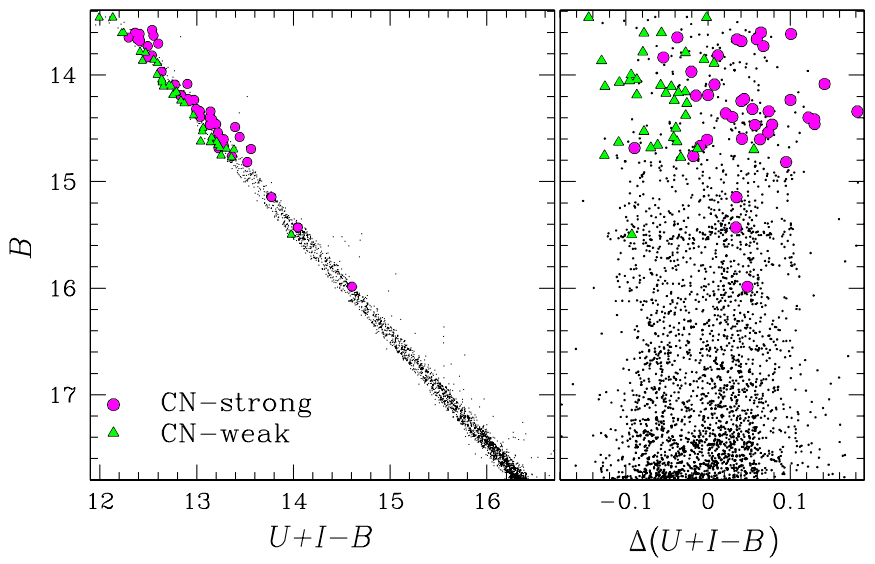

Figure 23. $\mathrm{CN}$-strong and $\mathrm{CN}$-weak stars defined in Figure 22, marked in our $B$ vs. $U+I-B$ plane (left panel) and $B$ vs. $\Delta(U+I-B)$ plane (right panel). (A color version of this figure is available in the online journal.)

both the "N-poor" and "N-rich" stars we assumed $[\mathrm{Fe} / \mathrm{H}]=$ -0.75 and $[\alpha / \mathrm{Fe}]=0.4$. The spectra were then integrated over the transmission of HST filters F275W, F336W, F390W, and F435W to derive the fluxes expected in those bands.

Figure 32 compares a pair of synthetic spectra (those corresponding to subgiant stars) and shows the transmissions of the filters. This figure shows that the differences between the 


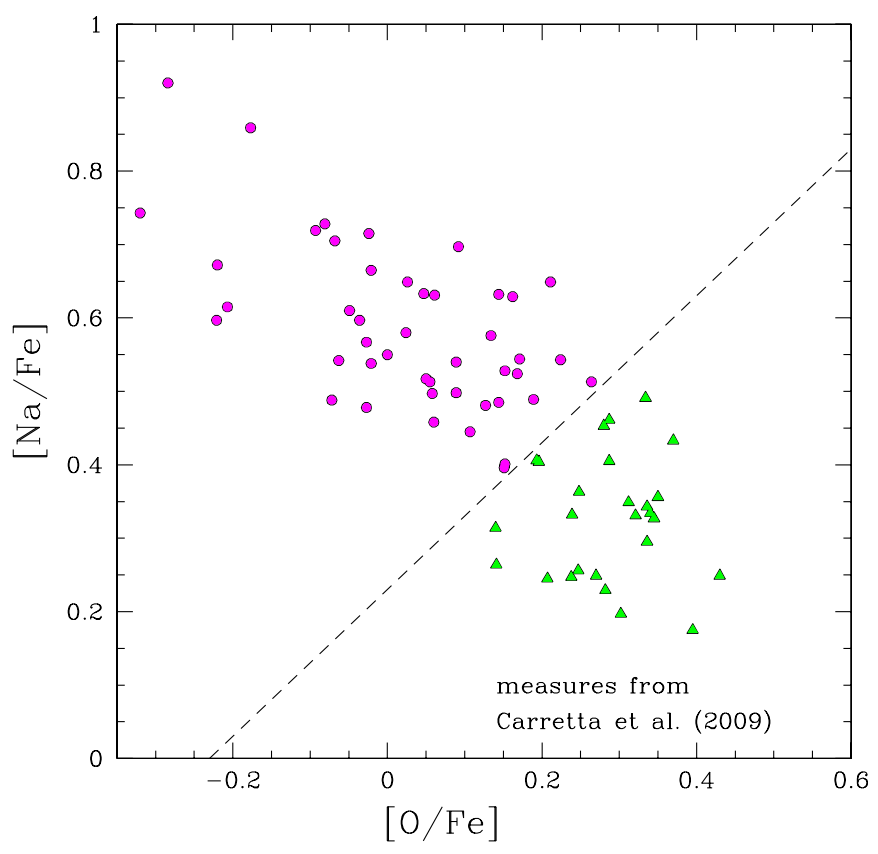

Figure 24. Sodium-oxygen anticorrelation for RGB stars from Carretta et al. (2009b). The dashed line arbitrarily separates Na-rich/O-poor stars (magenta circles) from Na-poor/O-rich stars (green triangles).

(A color version of this figure is available in the online journal.)

"N-poor" and "N-rich" spectra are essentially due to different strengths of the molecular bands. The $\mathrm{OH}$ band (in the wavelength range 2600-3200 $\AA$ ) is stronger in "N-poor" stars and falls within the F275W band; the NH band at $\sim 3400 \AA$ is stronger in the "N-rich" spectra and falls within the F336W band. The $\mathrm{CN}$ violet bands (stronger in N-rich spectra) at $3883 \AA$ and $4216 \AA$, and the $\mathrm{CH} G$ band (stronger in N-poor spectra) falls in the F390W band. The last two molecular bands are also within the $\mathrm{F} 435 \mathrm{~W}$ passband. As a consequence, the flux predicted for both the F336W and the F390W bands is smaller for the "N-rich" spectra than for the "N-poor" ones. The difference is greater for the F336W band, where it can be as much as $0.1 \mathrm{mag}$. The opposite holds for the F275W band. Abundance variations of $\mathrm{C}, \mathrm{N}, \mathrm{O}$ elements do not appreciably affect the stellar flux for passbands at longer wavelengths.

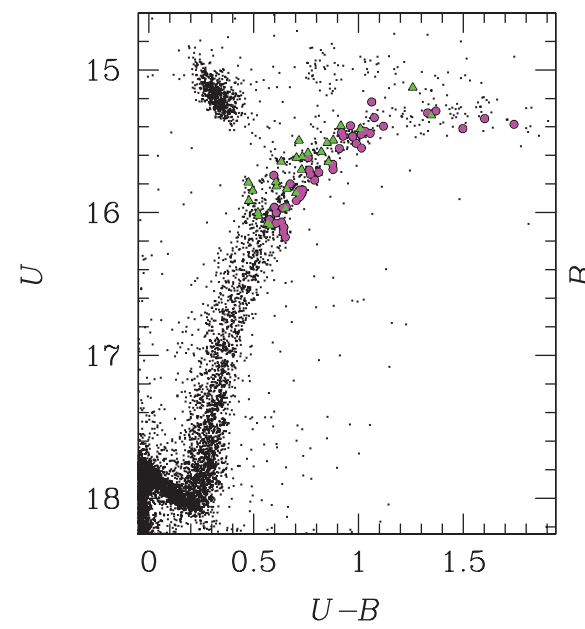

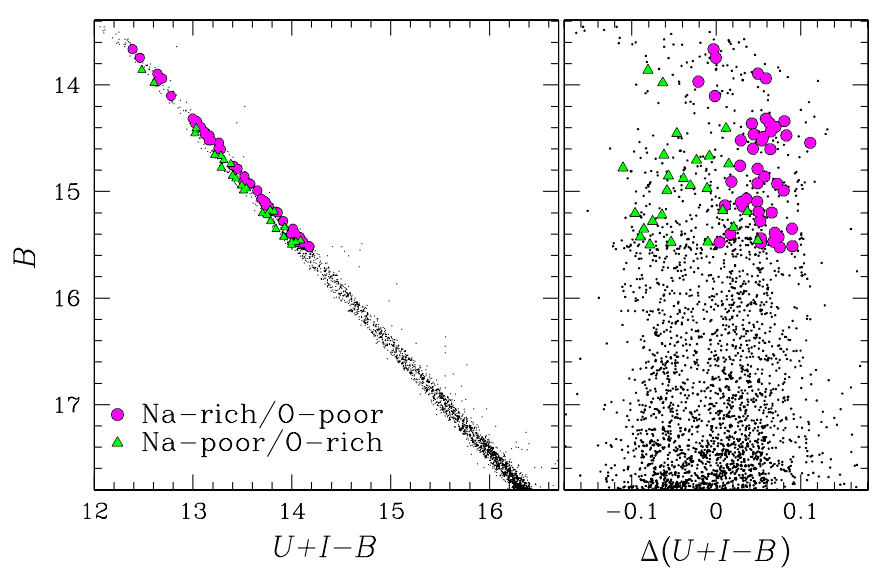

Figure 26. Na-poor/O-rich and Na-rich/O-poor RGB stars defined in Figure 24 are plotted here in $B$ vs. $U+I-B$ (left panel); the right-hand panel shows a verticalized version in our usual way.

(A color version of this figure is available in the online journal.)

We can now compare our theoretical values of three UV colors with the observed ones.

1. $m_{\mathrm{F} 275 \mathrm{~W}}-m_{\mathrm{F} 336 \mathrm{~W}}$. This color index is predicted to be larger (redder) for N-poor and smaller (bluer) for N-rich stars; this is the combined effect of less $\mathrm{OH}$ absorption in the $\mathrm{F} 275 \mathrm{~W}$ band and more $\mathrm{NH}$ absorption in the F336W band in N-rich compared to $\mathrm{N}$-poor stars. If $\mathrm{N}$ varies while $\mathrm{He}$ and $\mathrm{Mg}$ do not, the predicted color difference for the abundances given above is $0.17 \mathrm{mag}$ for the subgiant and $0.19 \mathrm{mag}$ for the red HB star.

2. $m_{\mathrm{F} 336 \mathrm{~W}}-m_{\mathrm{F} 435 \mathrm{~W}}$. This index is larger (redder) for the $\mathrm{N}$-rich and smaller (bluer) for the N-poor stars, again a result of stronger $\mathrm{NH}$ absorption in the $\mathrm{F} 336 \mathrm{~W}$ band. If there is again no difference in $\mathrm{He}$ and $\mathrm{Mg}$, the predicted color difference is $0.09 \mathrm{mag}$ for the subgiant and $0.10 \mathrm{mag}$ for the red HB star.

3. $m_{\mathrm{F} 390 \mathrm{~W}}-m_{\mathrm{F} 435 \mathrm{~W}}$. This index is larger (redder) for the $\mathrm{N}$-rich and smaller (bluer) for the $\mathrm{N}$-poor star, again due to stronger $\mathrm{NH}$ and especially $\mathrm{CN}$ absorption in the F390W band, and less $\mathrm{CH}$ absorption in the $\mathrm{F} 435 \mathrm{~W}$ band. If there is no difference in $\mathrm{He}$ and $\mathrm{Mg}$, the predicted color difference is $0.05 \mathrm{mag}$ for the subgiant and $0.07 \mathrm{mag}$ for the red $\mathrm{HB}$ star.

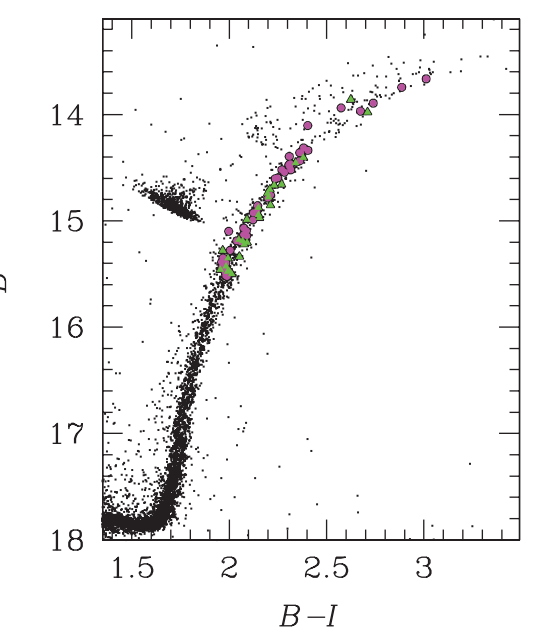

Figure 25. $U$ vs. $U-B$ (left panel) and $B$ vs. $B-I$ (right panel) CMD from ground-based photometry. The stars belonging to the two groups of Na-rich (O-poor) and Na-poor (O-rich) stars are represented with magenta circles and green triangles, respectively.

(A color version of this figure is available in the online journal.) 

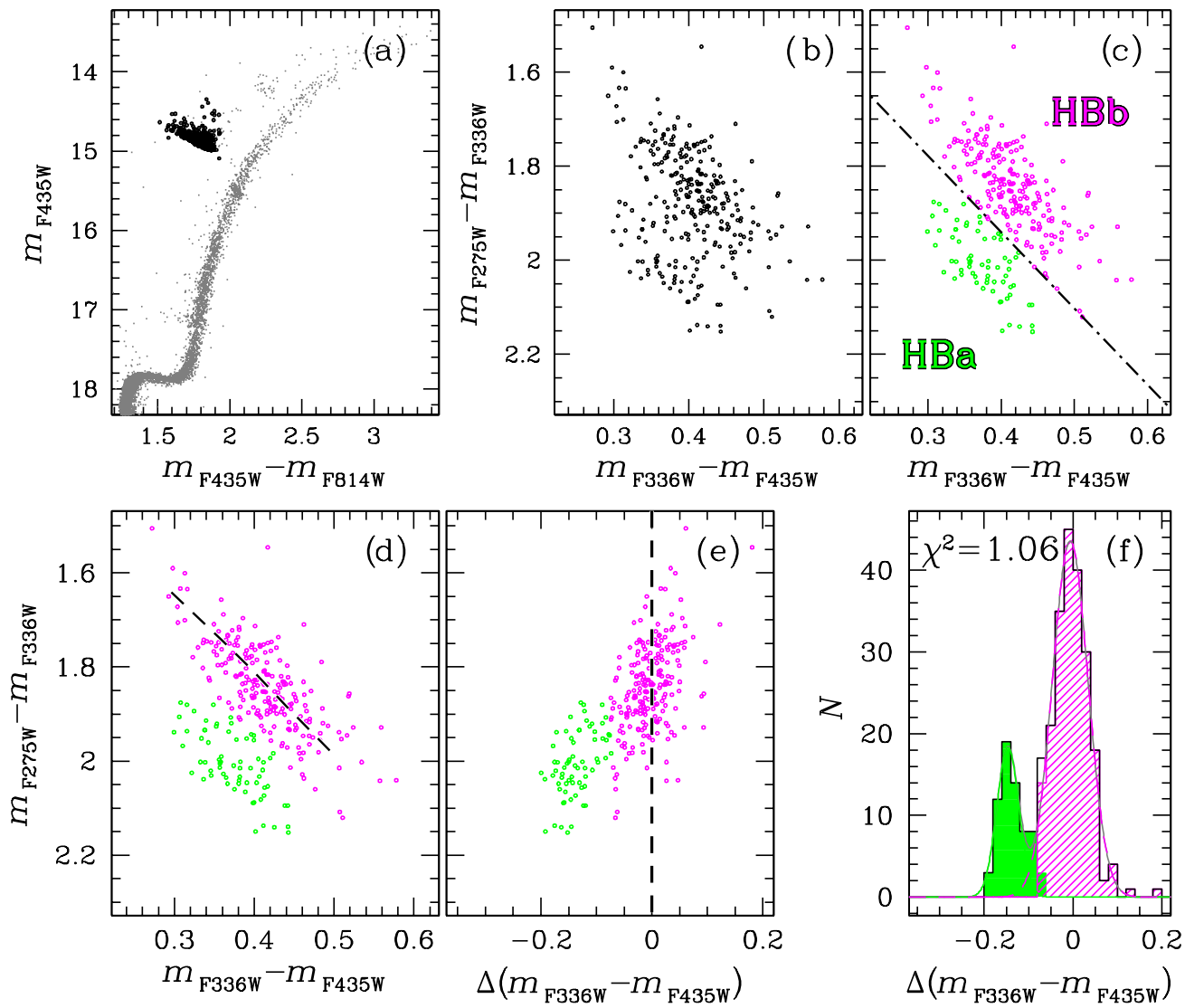

Figure 27. HB stars highlighted in black in panel (a) can be seen to have a bimodal distribution in the two-color diagram of panel (b), and in the next panel they are separated into two components. In the bottom row of panels their distribution in color is fitted with two Gaussians; see the text for details.

(A color version of this figure is available in the online journal.)
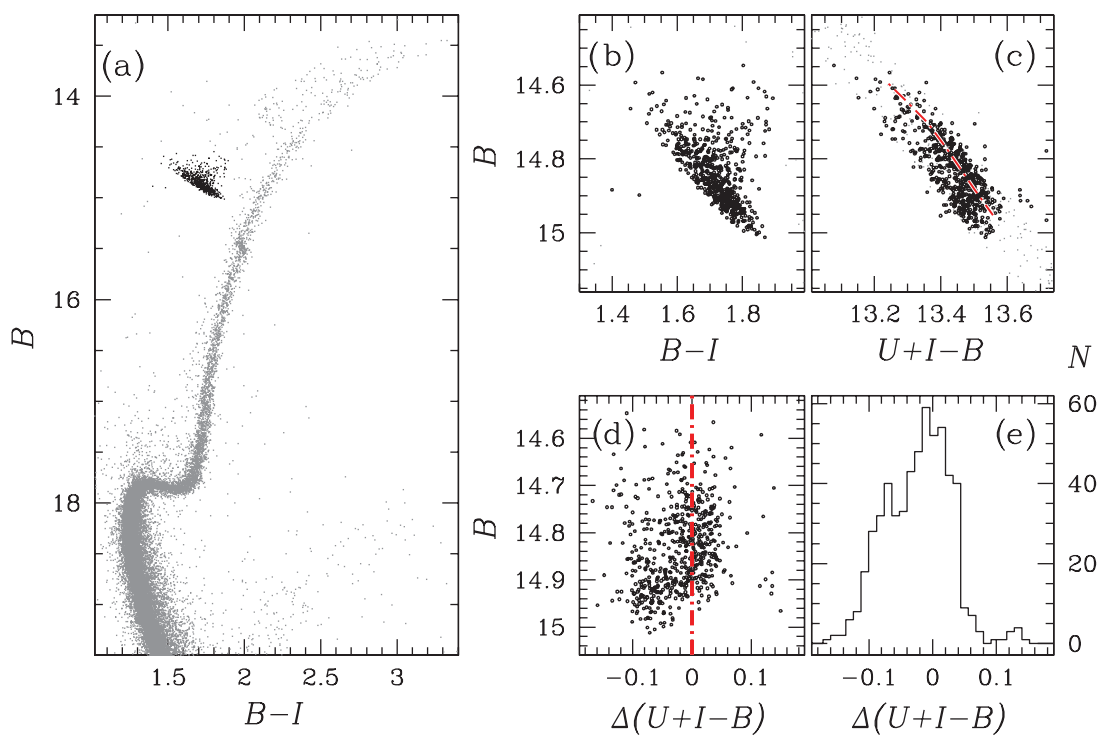

Figure 28. Panel (a): $B$ vs. $B-I$ CMD from ground-based photometry. HB stars are marked in black. A zoom of this CMD around the HB region is plotted in panel (b). Panel (c): HB stars in the $(B, U+I-B)$ plane; the red dashed line is the HBb fiducial drawn by hand. Panels (d) and (e) show the rectified $B$ vs. $\Delta(U+I-B)$ diagram and the histogram of the rectified colors.

(A color version of this figure is available in the online journal.)

These predicted differences are indeed similar to those observed in the two-color diagram in panels (b) and (c) of Figure 27.

It is of course a complication that additional differences are expected if the abundances of $\mathrm{He}$ and/or $\mathrm{Mg}$ are also different. The F275W band includes the very strong resonance doublet of
$\mathrm{Mg}$ II, so that the impact of $\mathrm{Mg}$ is appreciable (up to $\sim 0.05 \mathrm{mag}$ ). The main effect of a helium difference is a change in the effective temperatures of the stars: N-rich stars that are also He-rich are expected to be warmer and bluer. Even a small temperature difference has a quite dramatic effect on the UV bands: a difference of $100 \mathrm{~K}$ (corresponding to a change of $\sim 0.07$ in $Y$ ) 

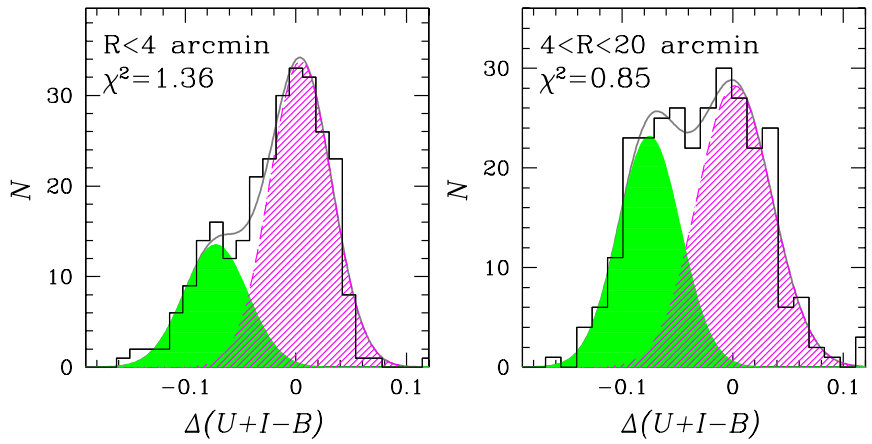

Figure 29. Histogram of the $\Delta(U+I-B)$ distribution of HB stars from groundbased photometry at two radial distances from the cluster center. (A color version of this figure is available in the online journal.)

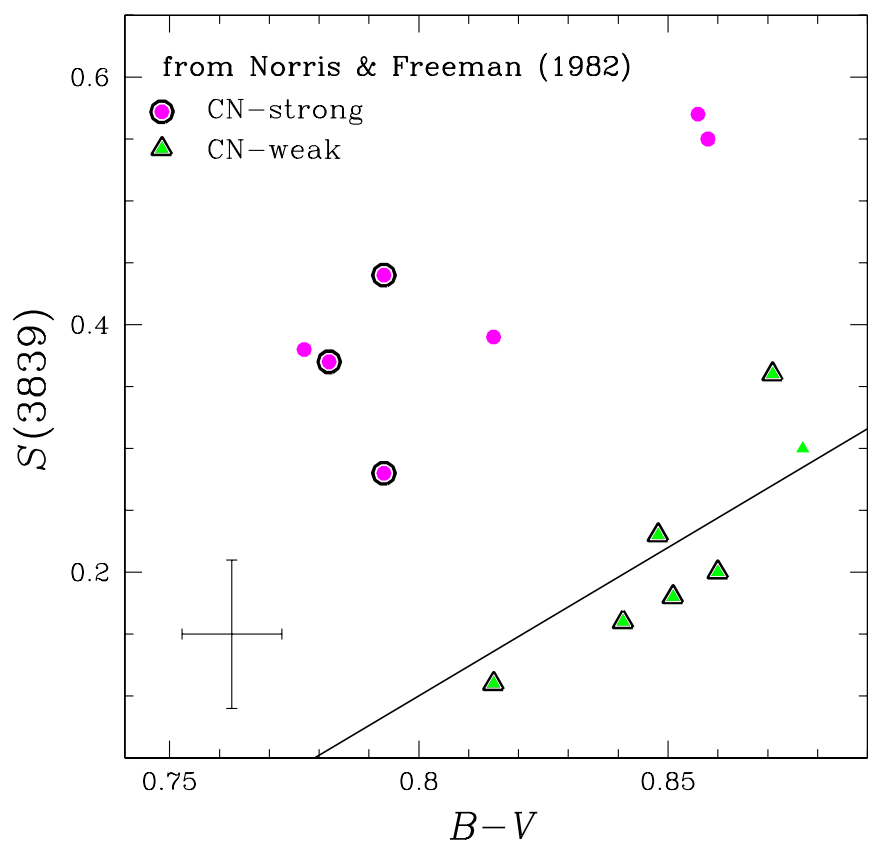

Figure 30. $S(3839)$ index for $14 \mathrm{HB}$ stars as a function of $B-V$ from Norris $\&$ Freeman (1982). The line shows the dependence of $S(3839)$ on the effective temperature as proposed by these authors. The $\mathrm{CN}$-weak and $\mathrm{CN}$-strong stars are plotted with green triangles and magenta circles, respectively, while we have marked with black contours the nine stars for which we have $U, B$, and $V$ photometry.

(A color version of this figure is available in the online journal.)

makes $m_{\mathrm{F} 275 \mathrm{~W}}-m_{\mathrm{F} 336 \mathrm{~W}}$ bluer by a further $\sim 0.2 \mathrm{mag}$, and more than offsets the difference in $m_{\mathrm{F} 336 \mathrm{~W}}-m_{\mathrm{F} 435 \mathrm{~W}}$ color between $\mathrm{N}$-rich and $\mathrm{N}$-poor stars. Such a large difference is clearly excluded by the photometry of 47 Tuc, which on the whole agrees quite well with only a minimal variation in helium $(\Delta Y \sim$ $0.015)$, as suggested in Section 4 in our attempt to explain the multicolor observations of the double MS.

\section{THE RADIAL DISTRIBUTION OF STELLAR POPULATIONS}

In the previous sections, we examined the radial gradients of the populations one sequence at a time. Here, we put all the information together to develop a comprehensive picture of the cluster. Since from an abundance perspective, most studies have focused on $\mathrm{CN}$, we will frame the discussion in terms of that molecule.

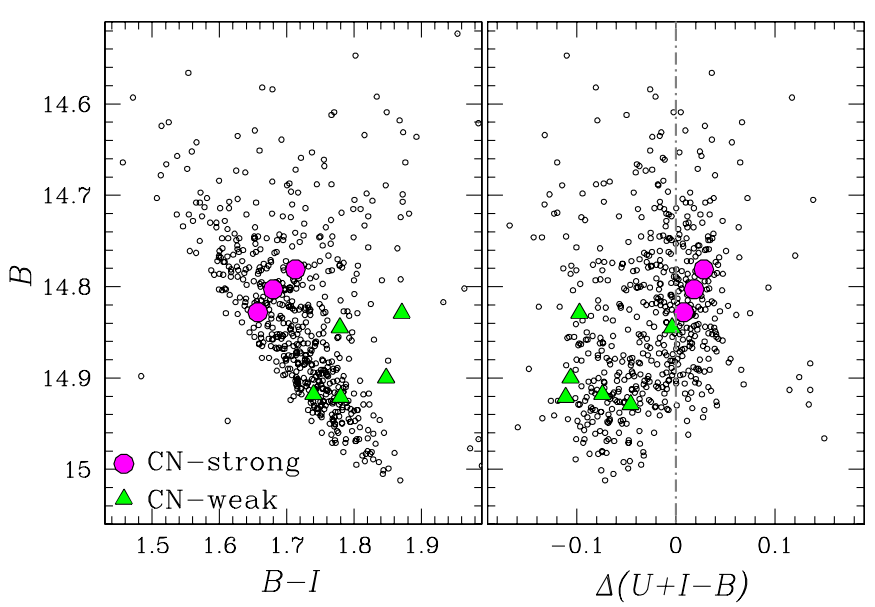

Figure 31. Reproduction of the $B$ vs. $B-I$ CMD (left panel) and the $B$ vs. $\Delta(U+I-B)$ diagram (right panel) for HB stars of Figures 28(b) and (d). The two groups of $\mathrm{CN}$-weak and $\mathrm{CN}$-strong stars as defined in Figure 30 are plotted with blue triangles and red circles.

(A color version of this figure is available in the online journal.)

The spatial distribution of stellar populations with different $\mathrm{CN}$ in 47 Tuc has been widely studied and debated in the literature, and little doubt remains concerning the presence of a significant radial gradient. On the basis of $\mathrm{CN}$ measurements of 142 RGB stars Norris \& Freeman (1979) found that the $\mathrm{CN}$-strong population is more centrally concentrated. But the same data were further analyzed by Hartwick \& McClure (1980), who found no evidence of differences in radial distribution of stars with different CN strength. Norris \& Smith (1981) found $\mathrm{CN}$-strong stars in the majority in the inner $\sim 3$ arcmin, a nearly equal fraction of $\mathrm{CN}$-strong and $\mathrm{CN}$-weak stars between $\sim 3$ and $\sim 15$ arcmin, and a predominance of $\mathrm{CN}$-weak stars at larger radial distances. Langer et al. (1989) concluded that because of the small sample size the results of Norris \& Freeman (1979) should be considered inconclusive. Finally, Briley (1997) studied the radial distribution of the CN-strong and $\mathrm{CN}$-weak populations on the basis of $\sim 300 \mathrm{RGB}$ stars with radial distances larger than 4 arcmin. He found that the relative numbers of $\mathrm{CN}$-rich and $\mathrm{CN}$-poor stars are roughly constant within $\sim 13$ arcmin of the cluster center, while the distribution exterior to 13 arcmin is clearly biased toward CN-poor stars, thus confirming the radial gradient first detected by Norris \& Freeman (1979).

An obvious advantage of photometric measurements is that we can study more stars and therefore get better statistics. In the following, we take advantage of the large size of our photometric catalogs to analyze the radial distributions of the multiple stellar sequences along the RGB and the HB. In Section 6, we used both HST and ground-based photometry to estimate the RGB population ratio in four radial intervals, while in Section 7 we determined the fraction of stars in the two HB segments at three radial distances.

The results for the radial distributions of HB and RGB stars are summarized in Figure 33, where we have plotted the fraction of $\mathrm{HBb}$ with respect to the total HB stars (green triangles) and the fraction of RGBb stars with respect to the total number of RGB stars (red dots). For completeness we also show (as a blue square) the fraction of MSb stars with respect to the total number of MS stars. (The MS split could be measured only at the center, where we have deep HST images; the ground-based images are too shallow and crowded to allow the MS populations to be discerned.) We find that in the central field the fraction of MSb, 

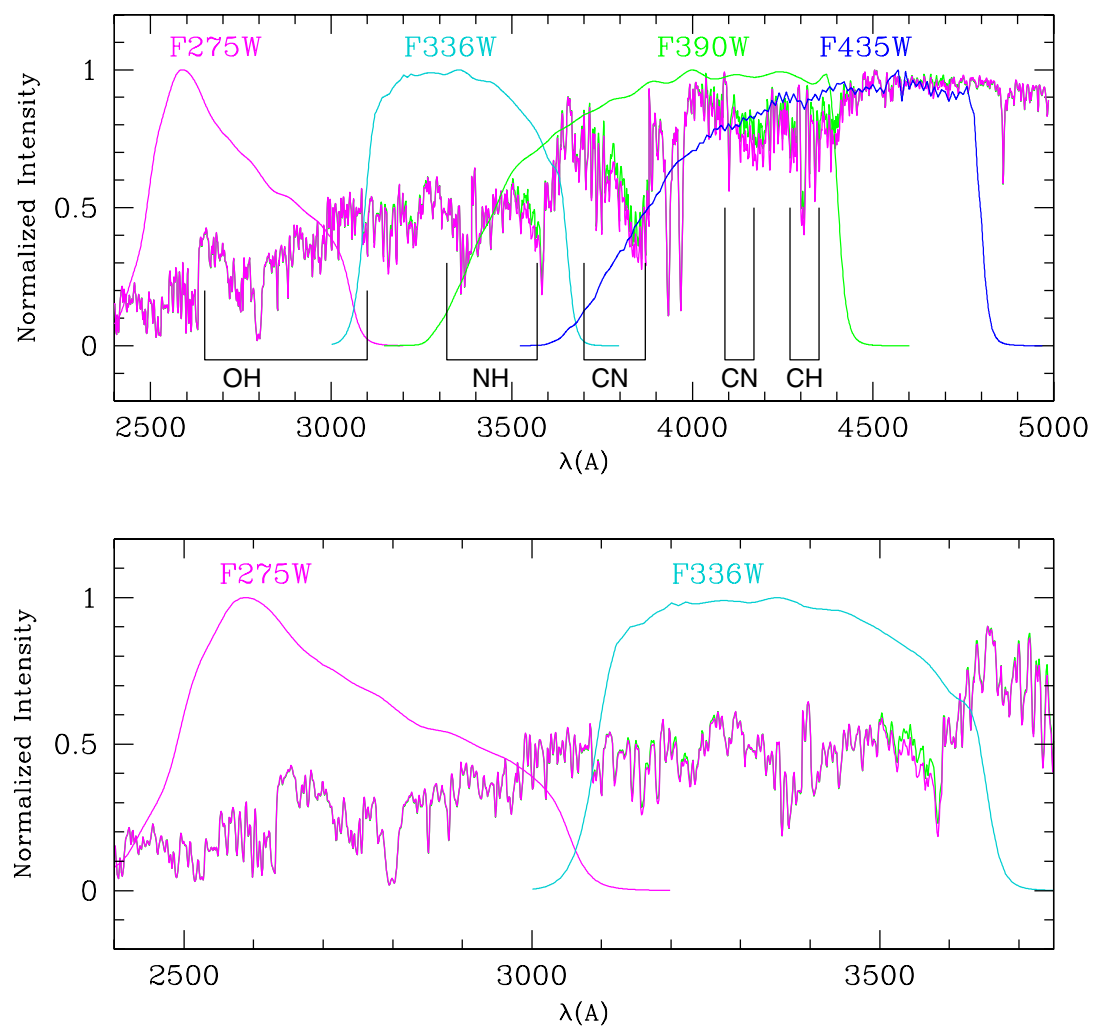

Figure 32. Top panel: comparison between two synthetic spectra: one for an N-rich star (magenta) and one for an N-poor star (black). The spectra are given as flux (in arbitrary units) and are smoothed at 1 Å resolution for clarity; they have been computed for parameters typical of a subgiant star in 47 Tuc, with chemical compositions given in the text. For reference, the normalized throughputs of the bluest broadband filters of WFC3/UVIS F(275/336/390/435/475)W are also shown. Labels on the bottom indicate the wavelength range where important spectroscopic features involving CNO elements cause significant absorption. The most important contributions come from $\mathrm{OH}$ at $\sim 2600-3100 \AA$ and NH at $\sim 3300-3600 \AA$. Bottom panel: a zoom-in of the spectral region that is of particular interest for the present paper.

(A color version of this figure is available in the online journal.)

$\mathrm{RGBb}$, and $\mathrm{HBb}$ stars with respect to the total number of MS, RGB, or HB stars, respectively, is about $80 \%-82 \%$ for each. For the RGB and HB this fraction falls to about $60 \%$ in the outer parts of the cluster. Thus the RGBb and $\mathrm{HBb}$ populations, which likely represent a second generation, appear to be more centrally concentrated. An integration of the $a / b$ population ratio, adopting a King model appropriate for 47 Tuc, reveals that globally the first generation (MSa, SGBa, RGBa, $\mathrm{HBa}$ ) accounts for $\sim 30 \%$ of the present-day stellar content of the cluster, while the second generation (MSb, SGBb, RGBb, HBb) accounts for the $\sim 70 \%$ majority share of the cluster, in agreement with the fraction of first- and second-generation stars measured by Carretta et al. (2009b) on the basis of the $\mathrm{Na}$ and $\mathrm{O}$ content. One final note it should be remembered that the proportions that we have quoted refer only to the sum of the components that we have called $a$ and $b$, which together make up only $92 \%$ of the total, the other $8 \%$ being the third population, which we glimpse only as the faint component of the SGB. In the other regions of the CMD the third population presumably makes up some small fraction of the parts that we call $a$ and $b$.

Such a global predominance of the second generation sets strong constraints on scenarios for the formation of multiple populations in GCs, and actually for the formation of GCs tout court. The fact that the second generation is much more centrally concentrated has also been observed in other GCs (such as $\omega$ Centauri; Sollima et al. 2007; Bellini et al. 2009) and suggests that much of the first generation (whatever it was) might have been tidally stripped from the progenitor of 47 Tuc. This is consistent with a similar suggestion for $\omega$ Cen by Bekki \& Norris (2006). We note that hydrodynamic plus $N$-body simulations of

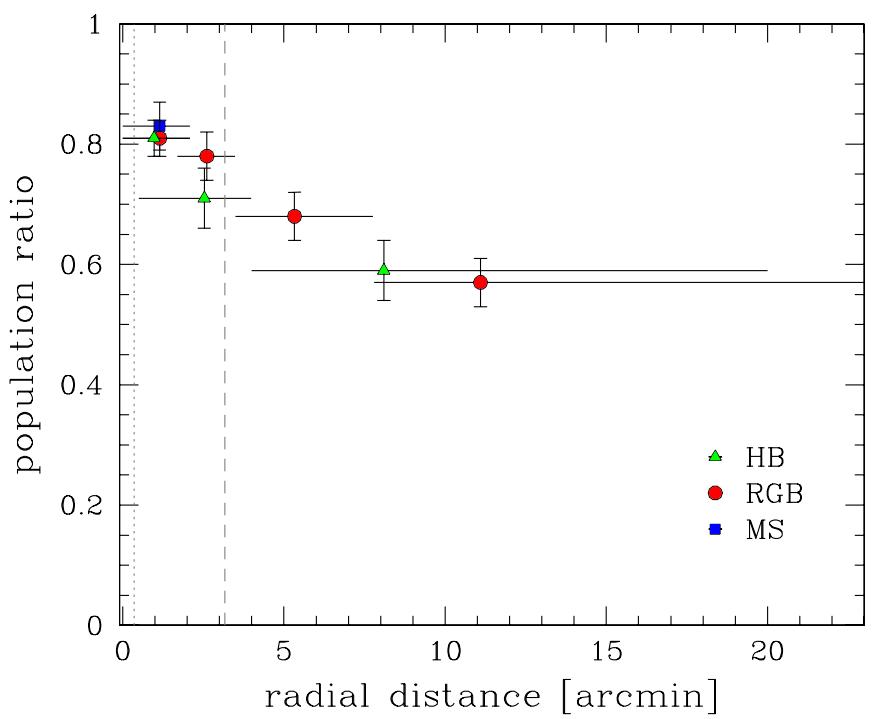

Figure 33. Radial distribution of the fraction of $\mathrm{HBb}$ (green triangles), RGBb (red circles), and MSb stars (blue square) with respect to the total number (component $\mathrm{a}+$ component $\mathrm{b}$ ) of HB, RGB, and MS stars, respectively. The horizontal lines indicate the radial extent of the region corresponding to each measure. Vertical dotted and dashed lines mark the core and the half-mass radius, respectively.

(A color version of this figure is available in the online journal.)

the formation of multiple stellar populations in GCs predict a larger concentration of second-generation stars in the cluster central regions (D'Ercole et al. 2008; Decressin et al. 2008). 

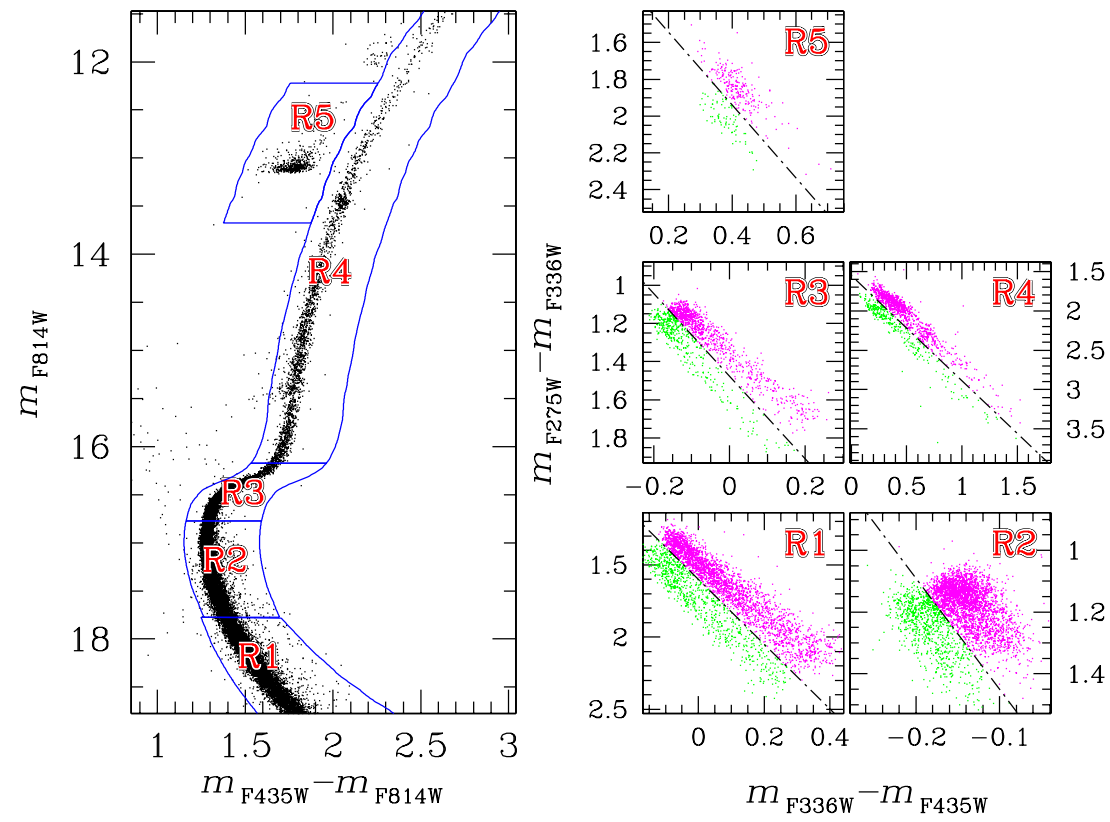

Figure 34. Left: $m_{\mathrm{F} 814 \mathrm{~W}}$ vs. $m_{\mathrm{F} 435 \mathrm{~W}}-m_{\mathrm{F} 814 \mathrm{~W}} \mathrm{CMD}$ from $H S T$ photometry, used to define the five regions labeled R1, R2, .., R5. Right: $m_{\mathrm{F} 275 \mathrm{~W}}-m_{\mathrm{F} 336 \mathrm{~W}} \mathrm{vs}$ $m_{\mathrm{F} 336 \mathrm{~W}}-m_{\mathrm{F} 435 \mathrm{~W}}$ two-color diagrams for stars in the five CMD regions. Dash-dotted lines are used to arbitrarily separate the two sequences that are present in each part of the CMD.

(A color version of this figure is available in the online journal.)
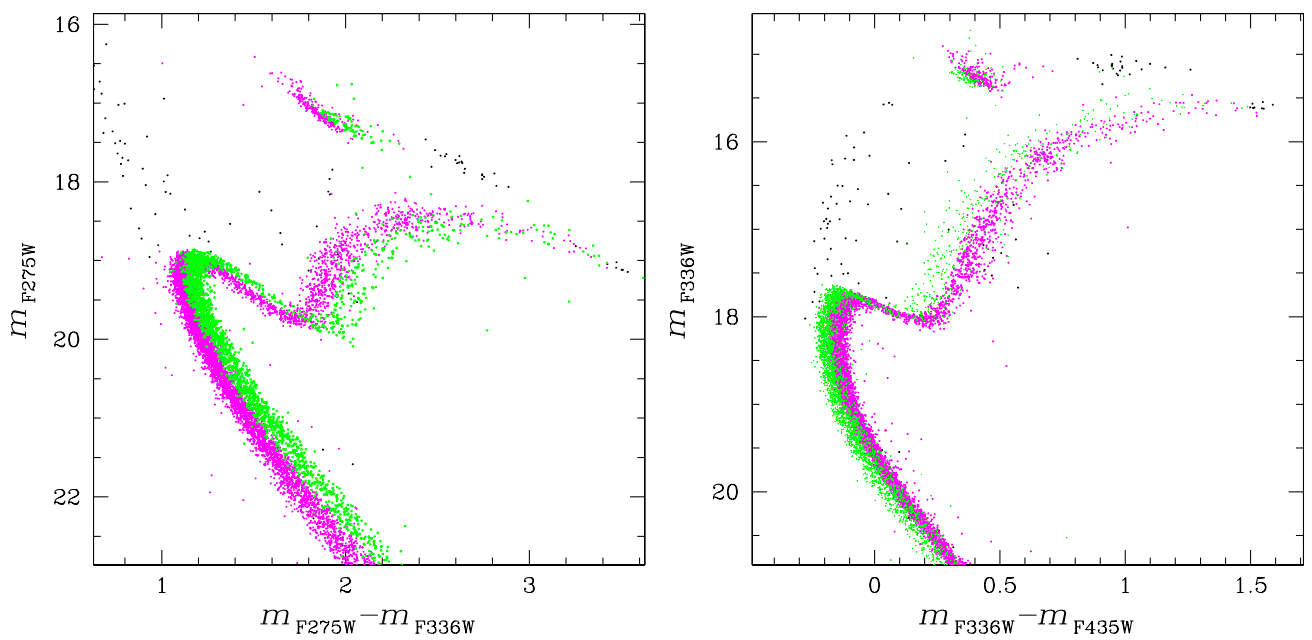

Figure 35. CMDs with $m_{\mathrm{F} 275 \mathrm{~W}}$ vs. $m_{\mathrm{F} 275 \mathrm{~W}}-m_{\mathrm{F} 336 \mathrm{~W}}$ (left) and $m_{\mathrm{F} 336 \mathrm{~W}}$ vs. $m_{\mathrm{F} 336 \mathrm{~W}}-m_{\mathrm{F} 435 \mathrm{~W}}$ (right). We have colored in green and magenta the two groups of stars selected in Figure 34. This is the first time anyone has been able to follow two stellar populations in a GC from the main sequence to the HB.

(A color version of this figure is available in the online journal.)

\section{CONNECTING THE MULTIPLE SEQUENCES ALONG THE MS, SGB, RGB, AND HB}

So far we have analyzed each evolutionary phase separately from the others, using the $m_{\mathrm{F} 275 \mathrm{~W}}-m_{\mathrm{F} 336 \mathrm{~W}}$ versus $m_{\mathrm{F} 336 \mathrm{~W}}-m_{\mathrm{F} 435 \mathrm{~W}}$ two-color diagram to separate multiple stellar populations along each of the evolutionary phases: MS, SGB, RGB, and HB. The various CMDs show that in our central HST field the stellar distribution is bimodal for all such evolutionary phases, with about $20 \%$ of the stars of each phase falling in the lower-left part of the two-color diagram, and the remaining $80 \%$ populating the upper right part of the diagram.

This behavior is summarized in Figure 34, where we have used the CMD in the left panel to select five regions labeled $\mathrm{R} 1-\mathrm{R} 5$, respectively, marking the MS, the turn-off region, the $\mathrm{SGB}$, the RGB, and the HB. In the right panels are plotted the two-color diagrams for stars in each of these CMD regions, with the black dash-dotted lines drawn so as to separate the two groups of stars that are colored green and magenta, just as in all previous sections.

The behavior of the two groups is strikingly similar from the MS all the way to the HB. Each of these stages shows a similar $80 / 20$ ratio of star numbers in the two groups, in the central field, and a similarly decreasing radial trend of this ratio. The most straightforward interpretation is that we are seeing two populations that wind their way, along nearly parallel paths, through the various successive stages of stellar evolution. This continuity is pictured in Figure 35, whose two CMDs emphasize the crucial role that the F275W and F336W filters play in seeing such simplicity in what would otherwise have been a perplexing mélange of details. 
Table 3

Chemical Composition and Fraction of Stars Relative to the Total Number, for the Two Main Population Groups

\begin{tabular}{lcccc}
\hline \hline Group & Color Code & Sequences & Chemical Composition & $\begin{array}{c}\text { Fraction } \\
R<\sim 2 \text { arcmin }\end{array}$ \\
& & & $\begin{array}{c}\text { Fraction } \\
R>15 \text { arcmin }\end{array}$ & $\sim 20 \%$ \\
\hline a & Green & MSa+SGBa+RGBa+HBa & CN-weak, O-rich, Na-poor, $Y \sim 0.25$ & $\sim 40 \%$ \\
b & Magenta & MSb+SGBb+RGBb+HBb & CN-strong, O-poor, Na-rich, $Y \sim 0.265$ & $\sim 80 \%$ \\
\hline
\end{tabular}

Finally, the main properties of the two populations are summarized in Table 3.

\section{SUMMARY AND DISCUSSION}

We have analyzed a large set of $H S T$ and ground-based images of the Galactic GC NGC 104 (47 Tuc) in nine photometric bands, finding multiple sequences throughout the various CMDs, from the MS all the way to the HB. Exploiting this wealth of HST data to investigate the behavior of the multiple populations as seen in several different combinations of magnitudes and colors, we found that among the rainbow of possible CMDs, those involving the F275W and F336W filters are particularly effective in separating components of otherwise entangled cluster populations. Taking a cue from $H S T$, we were able to construct a color system based on the $U$ band that exhibited a similarly effective separation of the populations in ground-based data.

We found that the distribution of stars along the MS, SGB, $\mathrm{RGB}$, and $\mathrm{HB}$ was in every case bimodal in the $m_{\mathrm{F} 275 \mathrm{~W}}-m_{\mathrm{F} 336 \mathrm{~W}}$ versus $m_{\mathrm{F} 336 \mathrm{~W}}-m_{\mathrm{F} 435 \mathrm{~W}}$ two-color diagram, and we finally put together the groups of stars that we had separated in this way, so as to draw a continuous connection between their successive evolutionary phases.

Near the cluster center all evolutionary phases split into two near-parallel sequences, with the richer one making up about $80 \%$ of the cluster stars, and the poorer one the remaining $20 \%$. Wide-field ground-based photometry allowed us to identify and separate the two sub-populations at larger radial distances from the cluster center, with the result that the majority population is more centrally concentrated, but its relative fraction decreases outward and approaches 50/50 in the outskirts of the cluster. Globally, the majority population accounts for $\sim 70 \%$ of the whole population of 47 Tuc, most of the remainder consisting of the minority population. Radial gradients in the stellar populations of this cluster have been known for a long time, with a $\mathrm{CN}$-strong population more centrally concentrated than the $\mathrm{CN}$-weak one. This suggests that the numerically dominant population is $\mathrm{CN}$-strong.

Along these same lines, we used $\mathrm{CN}$ band strengths and $\mathrm{Na}$ and $\mathrm{O}$ abundances that are available from the literature for some RGB and HB stars of both populations to investigate their chemical content. It appears that the more populous $\mathrm{RGBb}$ and $\mathrm{HBb}$ sequences consist of $\mathrm{CN}$-strong/Na-rich/O-poor stars, while the bulk of the $\mathrm{CN}$-weak/Na-poor/O-rich stars belong to the numerically poorer RGBa and $\mathrm{HBa}$.

On the theoretical side, we calculated synthetic spectra of MS stars with different chemical compositions, derived the corresponding colors for our filter set, and compared them with the observed colors in the two distinct populations. The colors of the minority population are well reproduced by stars with primordial helium abundance and an oxygen-rich/nitrogen-poor composition that is typical of halo stars of metallicity similar to that of 47 Tuc. On the other hand, the colors of the majority population stars are well reproduced by a composition in which nitrogen is greatly enhanced, along with a slightly increased helium, while carbon and oxygen are depleted. Synthetic spectra for RGB and HB stars confirm this result.

The most straightforward interpretation of these differences is that the minority population is the remnant of the first stellar generation, which formed out of the interstellar medium of its time, and shared its chemical composition. The chemical composition of the majority population, by contrast, carries the signatures of $\mathrm{CNO}$ and proton-capture processing at high temperatures, such as depletion of oxygen in favor of nitrogen and sodium, accompanied by helium enhancement. Therefore, the majority population should be regarded as the second stellar generation of 47 Tuc, which formed out of material that had been partly processed through stars of the first generation.

Since both populations share the same iron abundance, one can exclude from the enrichment history of the secondgeneration material any significant contribution by massive stars, exploding as core-collapse supernovae. This leaves intermediate-mass stars of the first generation as the obvious candidates for having processed the material that is now incorporated into the second generation, a view that is indeed widely entertained in the literature. Furthermore, it is certainly striking that the second generation is today more populous than the first. This sets strong constraints on the nature of the progenitor of the present cluster 47 Tuc, and on the initial mass of the first generation, which must have been one to two orders of magnitude more massive than the portion that is still bound to the cluster.

Finally, we must again remind the reader that besides the two populations that we have called first and second, we have found unmistakable evidence for the presence of a third population, including some $\sim 8 \%$ of the stars, which we can distinguish only on the SGB, but it appears to have the abundance distribution of the "b" population. This remains as a strident reminder that the stellar populations in 47 Tuc are more complex than the two-generation picture that we have just sketched.

A.P.M., G.P., A.B., R.G., S.C., A.R., A.B., E.C., F.D., M.D.C., S.L., and A.P. acknowledge partial support by PRIN MIUR 20075TP5K9 and PRIN INAF Formation and Early Evolution of Massive Star Clusters. G.P., S.C., and A.R. acknowledge partial support by ASI under the program ASI-INAF I/016/ 07/0. J.A. and I.R.K. acknowledge support from STScI grant GO-12311.

\section{REFERENCES}

Albrow, M. D., Gilliland, R. L., Brown, T. M., et al. 2001, ApJ, 559, 1060 Anderson, A. J. 1997, PhD thesis, Univ. California, Berkeley

Anderson, J., Bedin, L. R., Piotto, G., Yadav, R. S., \& Bellini, A. 2006, A\&A, 454, 1029

Anderson, J., \& King, I. R. 2006, Instrument Science Report ACS, 2006-01, 1 Anderson, J., Piotto, G., King, I. R., Bedin, L. R., \& Guhathakurta, P. 2009, ApJ, 697, L58

Anderson, J., Sarajedini, A., Bedin, L. R., et al. 2008, AJ, 135, 2055

Bedin, L. R., Cassisi, S., Castelli, F., et al. 2005, MNRAS, 357, 1038

Bedin, L. R., Piotto, G., Anderson, J., et al. 2004, ApJ, 605, L125

Bekki, K., \& Norris, J. E. 2006, ApJ, 637, L109 
Bell, R. A., Dickens, R. J., \& Gustafsson, B. 1975, BAAS, 7, 535 Bellini, A., Anderson, J., \& Bedin, L. R. 2011, PASA, 123, 622 Bellini, A., \& Bedin, L. R. 2010, A\&A, 517, A34

Bellini, A., Bedin, L. R., Piotto, G., et al. 2010, AJ, 140, 631

Bellini, A., Piotto, G., Bedin, L. R., et al. 2009, A\&A, 507, 1393

Bergbusch, P. A., \& Stetson, P. B. 2009, AJ, 138, 1455

Bragaglia, A., Carretta, E., Gratton, R. G., et al. 2010, ApJ, 720, L41 Briley, M. M. 1997, AJ, 114, 1051

Cannon, R. D., Croke, B. F. W., Bell, R. A., Hesser, J. E., \& Stathakis, R. A 1998, MNRAS, 298, 601

Carretta, E., Bragaglia, A., Gratton, R., \& Lucatello, S. 2009a, A\&A, 505, 139

Carretta, E., Bragaglia, A., Gratton, R. G., et al. 2006, A\&A, 450, 523

Carretta, E., Bragaglia, A., Gratton, R. G., et al. 2009b, A\&A, 505, 117

Cassisi, S., Salaris, M., Pietrinferni, A., et al. 2008, ApJ, 672, L115

Castelli, F. 2005, Mem. Soc. Astron. Ital. Supp., 8, 25

Catelan, M., Valcarce, A. A. R., \& Sweigart, A. V. 2010, in IAU Symp. 266 , Star Clusters: Basic Galactic Building Blocks Throughout Time and Space, ed. R. de Grijs \& J. R. D. Lépine (Cambridge: Cambridge Univ. Press), 281

D’Antona, F., Bellazzini, M., Caloi, V., et al. 2005, ApJ, 631, 868

D’Antona, F., \& Caloi, V. 2004, ApJ, 611, 871

D’Antona, F., Gratton, R., \& Chieffi, A. 1983, Mem. Soc. Astron. Ital., 54, 173

Decressin, T., Baumgardt, H., \& Kroupa, P. 2008, A\&A, 492, 101

Decressin, T., Meynet, G., Charbonnel, C., Prantzos, N., \& Ekström, S. 2007, A\&A, 464, 1029

de Mink, S. E., Pols, O. R., Langer, N., \& Izzard, R. G. 2009, A\&A, 507, L1

Denisenkov, P. A., \& Denisenkova, S. N. 1989, Astron. Tsirkulyar, 1538, 11

D’Ercole, A., Vesperini, E., D’Antona, F., McMillan, S. L. W., \& Recchi, S. 2008, MNRAS, 391, 825

Di Criscienzo, M., D’Antona, F., Milone, A. P., et al. 2011, MNRAS, 414, 3381

di Criscienzo, M., Ventura, P., D’Antona, F., Milone, A., \& Piotto, G. 2010, MNRAS, 408, 999

Freeman, K. C., \& Rodgers, A. W. 1975, ApJ, 201, L71

Gratton, R. G., Bonifacio, P., Bragaglia, A., et al. 2001, A\&A, 369, 87

Gratton, R. G., Bragaglia, A., Carretta, E., et al. 2003, A\&A, 408, 529

Gratton, R. G., Carretta, E., Bragaglia, A., Lucatello, S., \& D’Orazi, V. 2010, A\&A, 517, A81

Gratton, R., Sneden, C., \& Carretta, E. 2004, ARA\&A, 42, 385

Harbeck, D., Smith, G. H., \& Grebel, E. K. 2003, AJ, 125, 197

Harris, W. E. 1996, AJ, 112, 1487

Hartwick, F. D. A., \& McClure, R. D. 1980, ApJ, 235, 470

Hesser, J. E. 1978, ApJ, 223, L117

Hesser, J. E., Hartwick, F. D. A., \& McClure, R. D. 1977, ApJS, 33, 471

Kraft, R. P. 1979, ARA\&A, 17, 309

Kurucz, R. 1993, ATLAS9 Stellar Atmosphere Programs and 2 km/s grid, Kurucz CD-ROM No. 13 (Cambridge, MA: Smithsonian Astrophysical Observatory), 13

Kurucz, R. L. 2005, Mem. Soc. Astron. Ital., 8, 14

Landolt, A. U. 1992, AJ, 104, 340

Langer, G. E., Hoffman, R., \& Sneden, C. 1993, PASP, 105, 301
Langer, G. E., Shipman, R. F., \& Weida, M. J. 1989, PASP, 101, 811

Lardo, C., Bellazzini, M., Pancino, E., et al. 2011, A\&A, 525, A114

Lee, J.-W., Kang, Y.-W., Lee, J., \& Lee, Y.-W. 2009, Nature, 462, 480

Marino, A. F., Milone, A. P., Piotto, G., et al. 2009, A\&A, 505, 1099

Marino, A. F., Villanova, S., Milone, A. P., et al. 2011a, ApJ, 730, L16

Marino, A. F., Villanova, S., Piotto, G., et al. 2008, A\&A, 490, 625

Marino, A. F., et al. 2011b, A\&A, submitted

McClure, R. D., \& Osborn, W. 1974, ApJ, 189, 405

Milone, A. P., Bedin, L. R., Piotto, G., \& Anderson, J. 2009, A\&A, 497, 755

Milone, A. P., Bedin, L. R., Piotto, G., et al. 2008a, ApJ, 673, 241

Milone, A. P., Piotto, G., Bedin, L. R., \& Sarajedini, A. 2008b, Mem. Soc. Astron. Ital., 79, 623

Milone, A. P., Piotto, G., King, I. R., et al. 2010, ApJ, 709, 1183

Nataf, D. M., Gould, A., Pinsonneault, M. H., \& Stetson, P. B. 2011, ApJ, 736, 94

Norris, J. 1978, IAU Symp., The HR Diagram: The 100th Anniversary of Henry Norris Russell, ed. A. G. Davis Philip \& D. S. Hayes (Dordrecht: Reidel), 195

Norris, J. E. 2004, ApJ, 612, L25

Norris, J., \& Bessell, M. S. 1975, ApJ, 201, L75

Norris, J., \& Freeman, K. C. 1979, ApJ, 230, L179

Norris, J., \& Freeman, K. C. 1982, ApJ, 254, 143

Norris, J., \& Smith, G. H. 1981, in IAU Colloq. 68, Astrophysical Parameters for Globular Clusters, ed. A. G. Davis Phillip \& D. S. Hayes (Schenectady, NY: Davis), 109

Pietrinferni, A., Cassisi, S., Salaris, M., \& Castelli, F. 2004, ApJ, 612, 168

Pietrinferni, A., Cassisi, S., Salaris, M., \& Castelli, F. 2006, ApJ, 642, 797

Piotto, G. 2009, in IAU Symp. 258, The Ages of Stars, ed. E. E. Mamajek, D. R. Soderblom, \& R. F. G. Wyse (Cambridge: Cambridge Univ. Press), 233

Piotto, G., Bedin, L. R., Anderson, J., et al. 2007, ApJ, 661, L53

Piotto, G., Villanova, S., Bedin, L. R., et al. 2005, ApJ, 621, 777

Ramírez, S. V., \& Cohen, J. G. 2002, AJ, 123, 3277

Renzini, A. 1983, Mem. Soc. Astron. Ital., 54, 335

Renzini, A., \& Voli, M. 1981, A\&A, 94, 175

Sbordone, L. 2005, Mem. Soc. Astron. Ital., 8, 61

Sbordone, L., Bonifacio, P., \& Castelli, F. 2007, Proc. IAU Symp. 239, Convection in Astrophysics, ed. F. Kupka, I. W. Roxburgh, \& K. L. Chan (Cambridge: Cambridge Univ. Press), 71

Sbordone, L., Salaris, M., Weiss, A., \& Cassisi, S. 2011, A\&A, 534, A9

Sirianni, M., Jee, M. J., Benítez, N., et al. 2005, PASP, 117, 1049

Sollima, A., Ferraro, F. R., Bellazzini, M., et al. 2007, ApJ, 654, 915

Stetson, P. B. 2000, PASP, 112, 925

Stetson, P. B. 2005, PASP, 117, 563

Ventura, P., Caloi, V., D’Antona, F., et al. 2009, MNRAS, 399, 934

Ventura, P., \& D'Antona, F. 2009, A\&A, 499, 835

Woolley, R. V. D. R. 1966, R. Obs. Ann., 2, 1

Yong, D., Grundahl, F., D’Antona, F., et al. 2009, ApJ, 695, L62

Yong, D., Grundahl, F., Johnson, J. A., \& Asplund, M. 2008, ApJ, 684, 1159 